\title{
Diseño y Evaluación de Mezcladores de un Quemador Doméstico usando Gas Licuado de Petróleo
}

\author{
Freddy J. Rojas, Fernando O. Jiménez Ugarte, José A. Álvarez \\ Facultad de Ingeniería, Sec. Ing. Mecánica, Pontificia Universidad Católica del Perú, Av. Universitaria 1801, \\ San Miguel, Lima-Perú. (e-mail: fjrojas@pucp.edu.pe; ojimene@pucp.edu.pe; alexis.alvarez@pucp.pe)
}

Recibido Dic. 7, 2016; Aceptado Feb. 2, 2017; Versión final Abr. 10, 2017, Publicado Oct. 2017

\section{Resumen}

Se presenta el diseño y evaluación experimental de seis mezcladores para un quemador de premezcla de 1,5 kW de potencia térmica, usando gas licuado de petróleo como gas combustible. Se realiza una investigación centrada en los parámetros de diseño del mezclador para mejorar el desempeño de la cocina doméstica a gas en el proceso de combustión. Esto se manifiesta en el rendimiento térmico y las emisiones generadas. Se presenta el análisis de los mezcladores de aire-gas combustible a diferentes condiciones para determinar la más conveniente según el modelo. Se propone el desarrollo de una cocina de alta eficiencia y bajas emisiones, entregando a la entidad competente un proyecto de norma técnica en rendimiento térmico de cocinas domésticas a gas licuado de petróleo a nivel del mar.

Palabras clave: quemadores domésticos de GLP; rendimiento térmico; emisiones, diseño de cocinas

\section{Design and Evaluation of Mixers in Domestic Burner using Liquiefied Petroleum Gas}

\begin{abstract}
The design and experimental evaluation of six mixers for a premix burner of 1,5 kW thermal power, using liquefied petroleum gas as fuel gas is presented. An investigation focused on the design parameters of the mixer to improve the performance of the domestic gas cooker in the combustion process is developed. This is manifested in the thermal performance and the emissions generated, so the analysis of the air-fuel gas mixers is presented for different conditions to determine the most suitable according to the model. The construction of a high efficiency and low emissions cooker is proposed, delivering to the competent entity a project of a technical standard on thermal performance of domestic cookers with liquefied petroleum gas at sea level.
\end{abstract}

Keywords: LPG domestic burner; thermal performance; emissions, stove design 


\section{INTRODUCCIÓN}

En la actualidad, el consumo de combustible en el mundo se hace cada vez mayor, tal cual lo indica el estudio sobre las perspectivas de la evolución mundial en los ámbitos de la energía, la tecnología y la política climática (European Commission, 2003), según el cual, de mantenerse la tendencia en curso, la proyección de demanda energética hasta el año 2030 tendrá un crecimiento anual del $1.8 \%$. Asimismo, el predominio de los combustibles fósiles se mantendrá, lo que producirá mayores emisiones de $\mathrm{CO}_{2}$, incluso superior al consumo de energía (2.1\% anual por término medio), de modo que en el 2030 las emisiones mundiales serán más del doble que las registrada en el año 1990, Es por ello que es de importancia el desarrollo de investigaciones que permitan reducir el gasto de energía, así como darles mayor atención a los reglamentos del uso de combustibles (OSINERMING, 2014).

En Perú, el Ministerio de Energía y Minas, en búsqueda de concientizar acerca del buen uso de la energía, ha procedido con la elaboración de "Guías de Orientación del Uso Eficiente de la Energía y de Diagnóstico Energético", con las cuales se busca establecer los procedimientos y/o metodologías para orientar, capacitar, evaluar y cuantificar el uso racional de los recursos energéticos, para su aplicación en las distintas áreas de consumo de energía de nuestro país (Ministerio de Energía y Minas, 2008). En el caso del sector residencial, como lo indica una de las ya mencionadas "Guías de Orientación del Uso Eficiente de la Energía y de Diagnóstico Energético", en los hogares peruanos la cocina a gas es el equipo más representativo de consumo de energía térmica, por lo que si se busca mejorar su rendimiento se necesitará un adecuado análisis de los factores más influyentes.

Existen investigaciones que se enfocan en los diversos aspectos que pueden afectar el rendimiento térmico en un quemador de una cocina a gas. Un ejemplo de este es el realizado por Surange et al. (2014) quienes, a partir de los resultados de sus pruebas, encontraron que existe una correlación entre el área del cuello del tubo inyector y el área del quemador para obtener un mejor rendimiento, según la cual el primero debe ser un $43 \%$ del valor del segundo. En otros casos, se hizo énfasis en el uso de combustibles alternativos, como en la investigación realizada por Olubiyi et al. (2014), donde se construyó y evaluó un quemador con biogás, en el que se determinó el rendimiento de la cocina para transformar la energía del combustible en calor usando dos métodos: calentando agua (alcanzando un rendimiento térmico de $21 \%$ ) y cocinando arroz (rendimiento térmico de 60\%), concluyéndose que la distancia del inyector y el mezclador afecta la relación aire/gas, otorgando la posibilidad de mejorar el rendimiento mediante este método. Por otra parte, Namkhat y Jugjai (2010) estudiaron los efectos que puede producirse la temperatura del aire primario en la combustión y en la estructura de la flama en quemadores radiales atmosféricos, usando gas licuado de petróleo como combustible, observándose que el nivel de aire primario entrante, con o sin precalentamiento, está limitada por el diseño del mezclador y el quemador; y que el aire entrante al mezclador decrece al aumentar la temperatura del aire, debido a que el aire en el mezclador presenta mayor viscosidad, generando mayores emisiones debido a una combustión incompleta. Con respecto al quemador, se han realizado pruebas con un diseño compuesto de otros materiales; uno de la más resaltante es la desarrollada por Pantangi et al. (2011), quienes presentaron un modelo de quemador denominado quemador radial poroso, constituido en cinco partes: la zona de combustión, elaborada de carburo de silicio (SiC); una zona de precalentamiento, elaborada por esferas de alúmina $\left(\mathrm{Al}_{2} \mathrm{O}_{3}\right)$; una malla de alambre para sostener la zona de precalentamiento; una carcasa y un mezclador de forma cilíndrica hecho de teflón; con el cual se alcanzó $68 \%$ de rendimiento térmico, utilizando gas licuado de petróleo como combustible, como máximo. Por último, Gohil y Channiwala (2011) trabajaron en un estudio experimental para obtener los rendimientos térmicos en algunos quemadores de cocinas domesticas convencionales siguiendo un proceso similar al del presente estudio, obteniendo, como máximo, un rendimiento térmico de $66.27 \%$ para una potencia de 1.78 kW (2011).

En la presente investigación, se desarrolla un estudio experimental del funcionamiento del mezclador airegas para un quemador de premezcla doméstico; profundizando en aspectos tales como sus dimensiones, la velocidad y presión del abastecimiento del gas (Martínez, 1992; Jones, 1989). Es así que en búsqueda de desarrollar cocinas a gas (GLP) que posean un alto rendimiento térmico, se realizarán las pruebas correspondientes con distintos prototipos de mezclador. Por lo tanto, nuestro estudio contribuirá a la literatura actual a tener en cuenta el mejor modelo analizado y representado en los prototipos de mezcladores elaborados para conseguir resultados de mayor eficiencia y menor emisión de cocinas domésticas.

\section{MATERIALES Y MÉTODOS}

Para el análisis de los distintos mezcladores, se ha procedido con las pruebas de rendimiento de la cocina doméstica con los mezcladores incorporados, mediante la relación del calor absorbido durante el calentamiento de agua y el consumo térmico de la cocina empleada. 
El rendimiento del proceso se determina mediante la división entre la energía cedida al agua y la otorgada por el gas suministrado, de modo que se puede formular de la siguiente manera:

$\eta=\frac{E_{\text {agua }}}{E_{\text {gas }}} \times 100 \%$

Para calcular la energía proporcionada al agua se sigue lo establecido por la primera ley de la termodinámica; además, considerando que el líquido contenido en un recipiente se mantiene estático a lo largo del proceso, se le tratará como un sistema estacionario (Boles y Cengel, 2006). De esta manera se obtiene lo siguiente:

$\mathrm{E}_{\text {agua }}=\mathrm{m}_{\text {agua }} \cdot\left(\mathrm{u}_{\mathrm{f}}-\mathrm{u}_{\mathrm{i}}\right)$

Siendo $m_{\text {agua }}$ la masa de agua empleada para el ensayo; mientras que $u_{f}$ y $u_{i}$ representan la energía interna del agua al final y al inicio del calentamiento respectivamente. A su vez, se considera al agua contenida como una sustancia incompresible, pues se asume que se mantendrá constante el volumen específico (o densidad) de la sustancia, mientras que la presión permanecerá constante durante el incremento de la temperatura (Boles y Cengel, 2006). Por lo tanto, el valor de la energía cedida al agua se expresa como:

$E_{\text {agua }}=m_{\text {agua }} \cdot C_{\text {agua }}\left(T_{f}-T_{i}\right)$

Donde $C_{\text {agua }}$ representa el calor específico del agua a condiciones ambientales $\left(P_{a m b}=101.325 \mathrm{kPa}\right.$ y $\left.\mathrm{T}_{\mathrm{amb}}=20^{\circ} \mathrm{C}\right), \mathrm{T}_{\mathrm{f}}$ y $\mathrm{T}_{\mathrm{i}}$ los niveles de temperatura del agua al final y al inicio del ensayo respectivamente.

Por otro lado, la energía producida por el gas combustible se determina mediante la medición del caudal del flujo del gas que es expulsado a través del inyector y el tiempo que transcurre durante el calentamiento del agua. Asimismo, se debe tener en cuenta el poder calorífico inferior del combustible a utilizar. Siguiendo lo ya mencionado, se puede llegar a lo siguiente:

$E_{\text {gas }}=\dot{Q}_{\text {gas }} \cdot \rho \cdot t \cdot P C l$

Siendo $Q_{\text {gas }}$ el caudal del gas, cuyo valor puede variarse utilizando el regulador de flujo, pero se mantendrá el mismo nivel para todos los ensayos, con el fin de trabajar bajo las mismas condiciones; $\rho$ es la densidad del gas; $t$ representa el tiempo requerido para que, durante el ensayo, el agua contenida pase de la temperatura $\mathrm{T}_{\mathrm{i}}$ a $\mathrm{T}_{\mathrm{f}}$; y $\mathrm{PCl}$ como el poder calorífico inferior del gas combustible, el cual representa la cantidad de calor liberado cuando se quema por completo una unidad del combustible, mientras el agua resultante del proceso sale en forma de vapor en los gases de escape hacia el ambiente (Boles y Cengel, 2006)

Por último, cabe mencionar que para la realización de los ensayos se seguirán los pasos establecidos por la norma NCh927/1, elaborada por la División de Normas del Instituto Nacional de Normalización en Chile, la cual señala que el rendimiento obtenido debe ser mayor a $58 \%$ con PCl y $52 \%$ si se utiliza PCS (INNCHILE, 2001). Sin embargo, debe señalarse que se ha realizado un cambio con respecto a lo estipulado, modificando la temperatura de inicial del agua en los ensayos de $20^{\circ} \mathrm{C}$ a $25^{\circ} \mathrm{C}$. Los valores que se emplearon para las pruebas y cálculos se muestran en la Tabla 1.

Tabla 1: Valores de las variables para los ensayos

\begin{tabular}{|l|c|c|}
\hline \multicolumn{1}{|c|}{ Variable } & Unidad & Valor \\
\hline Masa de agua & $\mathrm{kg}$ & 3.439 \\
\hline Calor específico del agua & $\mathrm{kJ} / \mathrm{kg}{ }^{\circ} \mathrm{C}$ & 4.186 \\
\hline Temperatura inicial del agua & ${ }^{\circ} \mathrm{C}$ & 25 \\
\hline Temperatura final del agua & ${ }^{\circ} \mathrm{C}$ & 90 \\
\hline Temperatura del gas & ${ }^{\circ} \mathrm{C}$ & 21 \\
\hline
\end{tabular}

Es así que, siguiendo con lo estipulado por la norma NCh927/1, se ha de utilizar dos recipientes, cuyas dimensiones se encuentran ya establecidas, para calentar el agua. El primero es una cacerola hecha de aluminio con $22 \mathrm{~cm}$ de diámetro, esta contendrá $3.7 \mathrm{~kg}$ de agua y su función es la de precalentar el quemador antes de comenzar con los ensayos. El segundo es otra olla hecha de aluminio de $22 \mathrm{~cm}$ de diámetro, con la cual se realizaron las experiencias (INN-CHILE, 2001). Mientras que el proceso para el análisis de la emisión de monóxido de carbono neutro $\left(\mathrm{CO}_{\mathrm{n}}\right)$ consiste en hacer uso de una olla de $22 \mathrm{~cm}$ con $3,7 \mathrm{~kg}$ de agua y una campana extractora que posea las dimensiones indicadas en la norma NCh 927/1 (INN-CHILE, 2001). 
Las pruebas consisten en calentar el agua contenida en la olla, con la campana instalada sobre esta, de modo que dirija las emisiones de la combustión durante la ebullición a través de un tubo de cobre, en donde se colocará el analizador de gases. De modo que para estimar el contenido de monóxido de carbono (CO) referido a los productos de la combustión exentos de aire y vapor de agua (combustión neutra) se sigue la fórmula indicada en la ecuación (5):

$\mathrm{CO}_{\mathrm{n}}=\mathrm{CO}_{2 \mathrm{~N}} * \frac{\mathrm{CO}_{\mathrm{M}}}{\mathrm{CO}_{2 \mathrm{M}}}$

\section{En donde:}

$\mathrm{CO}_{\mathrm{n}}=$ Monóxido de carbono neutro (\%)

$\mathrm{CO}_{2 \mathrm{~N}}=$ Dióxido de carbono neutro calculado según el gas utilizado (\%)

$\frac{\mathrm{CO}_{\mathrm{M}}}{\mathrm{CO}_{2 \mathrm{M}}}=$ Relación de $\mathrm{CO}$ y $\mathrm{CO}_{2}$ medidos en las muestras obtenidas durante los ensayos de la combustión

En la Tabla 2 se indica los valores del porcentaje de dióxido de carbono neutro para los gases a usar durante las pruebas. Para esto se hará uso de un equipo analizador de gases KM9106 Quintox, el cual permite monitorear los gases de combustión generados por medio de una sonda con un eje de acero inoxidable, con una termocupla integrada.

Tabla 2: Gases y respectivos valores de $\left(\mathrm{CO}_{2}\right)_{\mathrm{N}} \%$ (INN-CHILE, 2001)

\begin{tabular}{|c|c|}
\hline Designación del gas & GLP \\
\hline$\left(\mathrm{CO}_{2}\right)_{\mathrm{N}} \%$ & 13.79 \\
\hline
\end{tabular}

\section{Combustible}

EI GLP es un hidrocarburo que se obtiene del proceso de refinamiento del petróleo, el cual está compuesto, para estas experiencias, de $40 \%$ de butano y $60 \%$ de propano. Está almacenado en un balón a presión, el cual se conecta al banco de pruebas mediante una manguera, un regulador y un juego de abrazaderas (OSINERGMING, 2014). EI GLP está categorizado como parte de la tercera familia de gases, la cual se caracteriza por estar conformada por gases cuyos constituyentes básicos son el propano y el butano (INNCHILE, 2001). Durante los ensayos con este combustible se trabajará con una presión de 28 mbar, pues este es el valor de operación común en las instalaciones a nivel residencial. De modo que los valores a utilizar para el cálculo de rendimiento se mostrarán en la Tabla 3:

Tabla 3: Datos del GLP (Rojas, 2016)

\begin{tabular}{|l|c|c|}
\hline Variable & Unidad & Valor \\
\hline Presión manométrica del flujo & $\mathrm{mbar}$ & 28 \\
\hline Caudal & $\mathrm{LPM}$ & 1,08 \\
\hline Poder Calorífico Inferior & $\mathrm{kJ} / \mathrm{kg}$ & 46090 \\
\hline Densidad & $\mathrm{kg} / \mathrm{m}^{3}$ & 2.1023 \\
\hline Relación de calores específicos $(\mathrm{k})$ & - & 1.135 \\
\hline Densidad Relativa (s) & - & 1.7161 \\
\hline Peso Molecular & $\mathrm{Kg} / \mathrm{kmol}$ & $17.7 \times 10^{-3}$ \\
\hline
\end{tabular}

\section{Sistema de Inducción}

Inyector: Durante los ensayos se utilizará un inyector que se encargue de dirigir el gas combustible hacia el mezclador, además permite convertir la energía potencial asociada a la alta presión del suministro de gas en la energía cinética del gas expulsado. Para la correcta elección del diámetro de salida de este elemento, se debe precisar si el gas descargado a través del orificio se dará a condiciones sónicas o subsónicas; para ello se calculará la relación de presión crítica a través de la siguiente ecuación:

$P_{c}=\left[\frac{2}{k+1}\right]^{\frac{k}{k-1}}$ 
Siendo k la relación de calores específicos del gas. En caso este valor sea mayor al cociente de la presión atmosférica entre la presión absoluta del gas, se concluirá que la salida del flujo es sónico; caso contrario, será subsónico. Teniendo en cuenta que las condiciones ambientales de trabajo están establecidas por las características de Lima, se obtiene que el flujo presenta un carácter subsónico (Baukal, 2003). Posteriormente, al estar el flujo de salida a condiciones subsónicas, se obtiene el diámetro del inyector requerido a través de la siguiente relación:

$\mathrm{A}_{\mathrm{l}}=\frac{\dot{\mathrm{m}}}{\mathrm{C}_{\mathrm{d}} \cdot \rho_{\mathrm{g} s} \cdot \mathrm{M}_{\mathrm{g}} \cdot \mathrm{C}_{\mathrm{e}}}$

Siendo $A_{t}$ el área de salida del inyector, $\dot{m}$ el flujo másico del gas, $C_{e}$ la velocidad de sonido del fluido, $M_{g}$ el número de Match del combustible, $\rho_{g s}$ la densidad del fluido a la salida del inyector y $C_{d}$ la constante de descarga del inyector (Baukal, 2003)

Se asumirá que el coeficiente de descarga es 1 , considerando así que el flujo de salida a través del inyector se dará en su totalidad. Posteriormente, a través de los resultados de los ensayos correspondientes, se estimará el valor del coeficiente de descarga " $\mathrm{C}_{d}$ " usando una versión empírica del teorema de Bernoulli:

$Q=0,0467 \cdot C_{d} \cdot A_{t} \cdot \sqrt{\frac{P_{g}}{s}}$

En donde $Q$ es el caudal del gas, $C_{d}$ es la constante de descarga, $P_{g}$ es la presión manométrica del gas y $\mathrm{S}$ es la densidad relativa del gas (Fulford, 1996).

Mezclador: Tal como su nombre lo indica, su función es la de permitir una correcta combinación del gas combustible con el aire, por lo que es de importancia reconocer qué aspectos influyen en su correcto diseño (Fulford, 1996).

Para el dimensionamiento del primer mezclador se aplicará lo recomendado por el autor Howard Neil Jones. Siendo la relación del área de salida del inyector $\left(A_{e}\right)$ y el área de la garganta del mezclador $\left(A_{g}\right)$ :

$\frac{A_{e}}{A_{g}}=\frac{s}{(s+1)(1+R)\left(1+C_{L}\right)}$

En donde "s" representa la densidad relativa del gas combustible, $\mathrm{R}$ puede tomar un valor adimensional entre 4 y 6 representando la proporción del aire primario y de la mezcla (lo cual es usual en quemadores domésticos) y $C_{L}$ es la suma de pérdidas individuales en las secciones de la garganta y el difusor del mezclador (Jones, 1989).

Para este caso, se ha decidido trabajar con $\mathrm{R}=6$. (Jones, 1989). Para el caso de $C_{L}$, este depende del largo del mezclador, del diámetro de la garganta y del ángulo de la sección del difusor. Es así que el promedio para el coeficiente de pérdidas en la sección de la garganta es de 0,10 a 0,20, Además, en el difusor, el mezclado

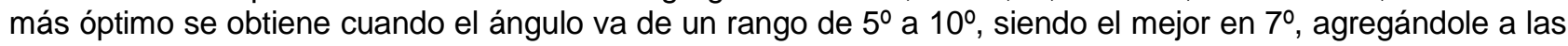
pérdidas un valor de 0,15 (Tabla 4). Por ende, en total, las pérdidas totales se pueden asumir desde 0,25 hasta 0,35 . Por lo tanto, se ha optado por utilizar un valor de 0,35 para $C_{L}$. (Martínez, 1992).

Tabla 4: Tabla de Pérdidas en la sección de difusor (Fuente: Jones, 1989)

\begin{tabular}{|l|c|c|c|c|c|}
\hline \multicolumn{6}{|c|}{ Coeficiente de pérdidas por fricción según el ángulo de la sección del difusor } \\
\hline Ángulo & $3^{\circ}$ & $5^{\circ}$ a $8^{\circ}$ & $10^{\circ}$ & $14^{\circ}$ & $20^{\circ}$ \\
\hline Coeficiente de pérdida & 0,18 & 0,14 & 0,16 & 0,25 & 0,45 \\
\hline
\end{tabular}

Para determinar las dimensiones del mezclador se ha de seguir lo mostrado en la Figura 1, donde se indica que el largo de las secciones está ligado al diámetro de la garganta ya calculado. Además, se señala que la reducción de la sección del difusor ocasiona una disminución en la proporción de aire en la mezcla de $2 \%$ (Martínez, 1992). Es así que se desarrollarán los dos modelos de mezcladores para cada gas combustible a emplear, correspondientes a la recomendación dada por la Figura 1. En los cuales se utilizó un ángulo de 7º para la sección divergente; mientras que para la entrada se usó un ángulo de $40^{\circ}$. 
Por otra parte, con el fin de analizar la influencia de la sección de entrada en la calidad de la combustión, se permitirá el desarrollo de un tercer mezclador, con las dimensiones mostradas en la Figura 2. Este mezclador, a diferencia de los dos primeros, posee una sección de entrada con el doble de largo de lo recomendado; manteniendo los demás aspectos; tales como el largo del difusor, así como los ángulos de las secciones de entrada y salida; iguales a los ya sugeridos para el primer mezclador.

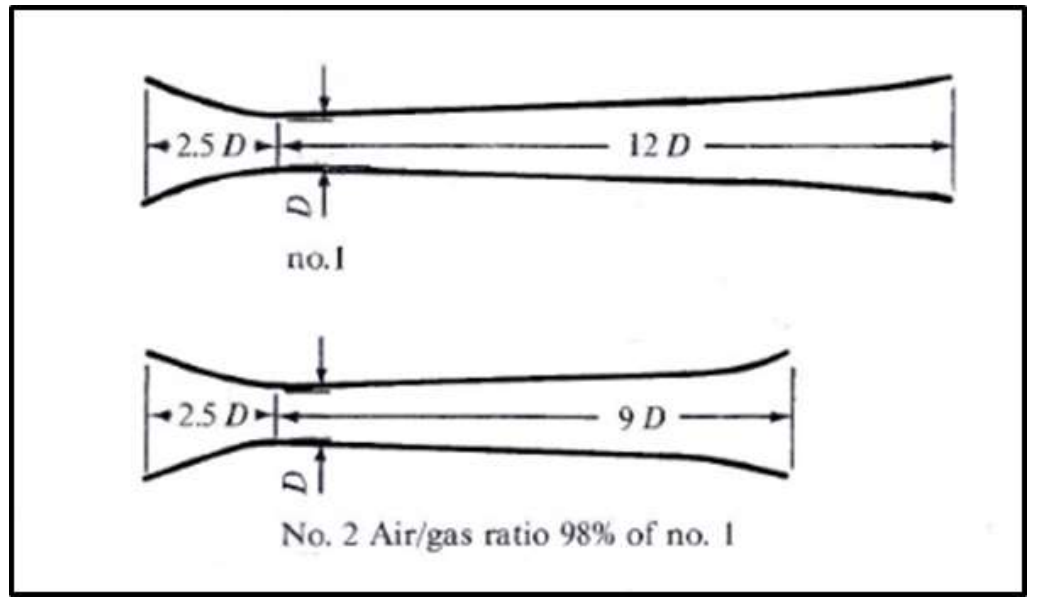

Fig. 1: Recomendaciones para el largo de los mezcladores Fuente: Jones, 1989

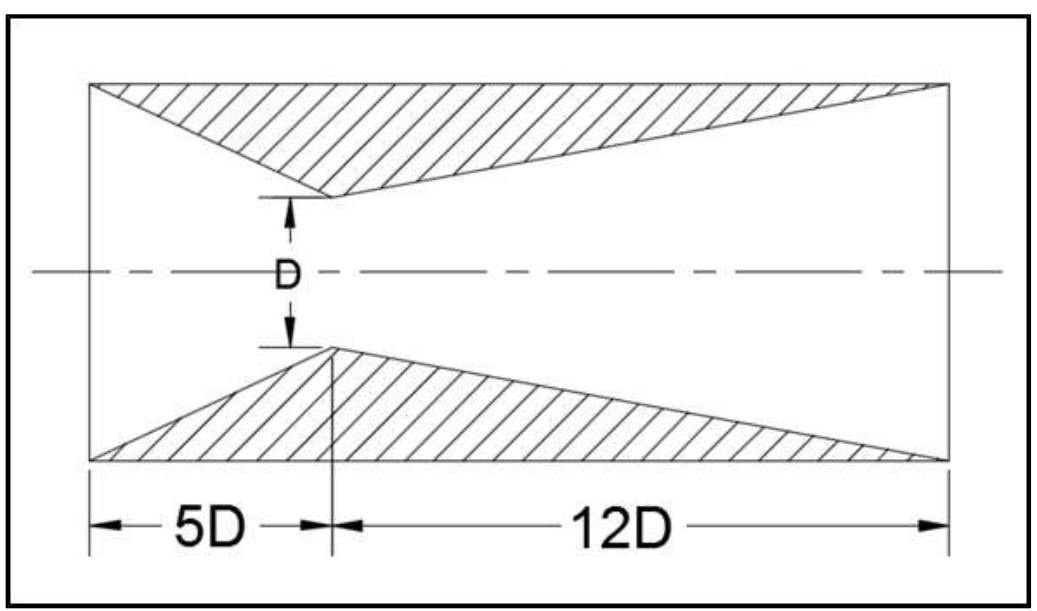

Fig. 2: Modelo de tercer mezclador (GLP-3)

Para el cuarto mezclador se seguirá lo recomendado por el autor Fulford (1996), para lo cual se procede con el análisis estequiométrico.

$\left(0,6 \mathrm{C}_{3} \mathrm{H}_{8}+0,4 \mathrm{C}_{4} \mathrm{H}_{10}\right)+5.6\left(\mathrm{O}_{2}+3.76 \mathrm{~N}_{2}\right) \rightarrow 3.4 \mathrm{CO}_{2}+4.4 \mathrm{H}_{2} \mathrm{O}+21,056 \mathrm{~N}_{2}$

Haciendo uso de un exceso de aire de $10 \%$ se obtiene lo siguiente:

$\left(0,6 \mathrm{C}_{3} \mathrm{H}_{8}+0,4 \mathrm{C}_{4} \mathrm{H}_{10}\right)+6.16\left(\mathrm{O}_{2}+3.76 \mathrm{~N}_{2}\right) \rightarrow 3.4 \mathrm{CO}_{2}+4.4 \mathrm{H}_{2} \mathrm{O}+23.1616 \mathrm{~N}_{2}+0,56 \mathrm{O}_{2}$

Es así que la relación de aire-gas combustible será la siguiente

$\mathrm{RAC}_{\mathrm{v}}=\frac{6.16 \times 4.76 \times 24.8}{1 \times 24.8}=29.322$

El monto de aire primario que será utilizado en la mezcla será de $50 \%$, tal como se recomienda en el paper "Biogas Stove Design", elaborado por Fulford (1996). De esta forma, la relación de arrastre está definida como:

$\mathrm{r}_{\mathrm{ar}}=0,5 \times \mathrm{RAC}_{\mathrm{v}}$ 
De esta manera, el diámetro de la garganta $\left(d_{g}\right)$ del mezclador tipo venturi se puede determinar a través de la expresión formulada por Prigg para un quemador ideal:

$d_{g}=\left(\frac{r_{a r}}{\sqrt{s}}+1\right) \times d_{i}$

Con respecto a la longitud de las secciones del mezclador, se seguirán las mismas recomendaciones que en la primera propuesta. Utilizando un ángulo de entrada de $40^{\circ}$ para la entrada convergente y un ángulo de salida de $7^{\circ}$ para la sección divergente.

El quinto modelo plantea el diseño de un mezclador que consiste solo en una entrada convergente (Figura 3), basado en un análisis realizado por medio del Software Ansys CFD (Cortes, 2009).

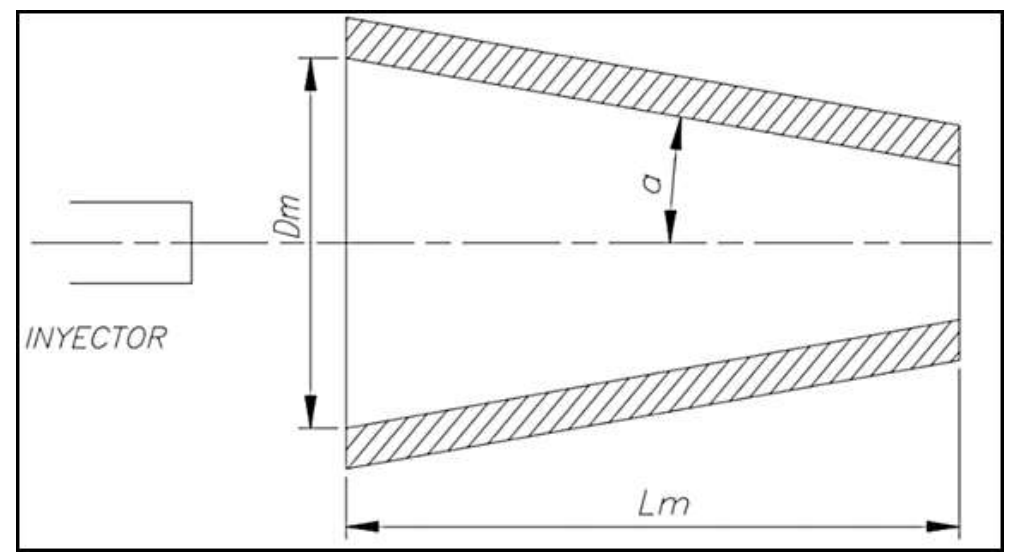

Fig. 3: Modelo esquemático del quinto mezclador

Tabla 5: Dimensiones del quinto modelo de mezclador

\begin{tabular}{|c|c|c|}
\hline $\begin{array}{c}\text { Longitud } \\
\text { "Lm" }(\mathrm{mm})\end{array}$ & $\begin{array}{c}\text { Ángulo de convergencia } \\
\text { "a" }\left({ }^{\circ}\right)\end{array}$ & $\begin{array}{c}\text { Diámetro de entrada } \\
\text { " } \mathrm{Dm} \text { " }(\mathrm{mm})\end{array}$ \\
\hline 50 & 13.8 & 26.05 \\
\hline
\end{tabular}

Para el desarrollo del sexto y último mezclador se seguirá lo planteado por el quinto modelo, en el aspecto

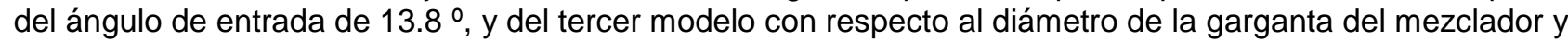
el largo de las secciones. Se muestra en la Figura 4 y la Tabla 6 una mejor visualización de las dimensiones de los mezcladores desarrollados.

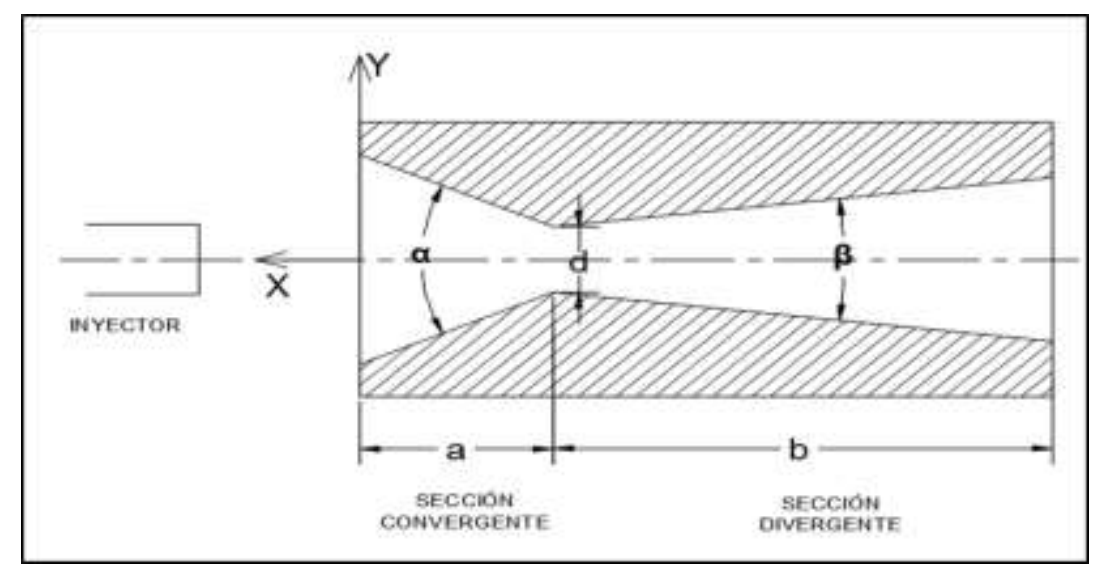

Fig. 4: Esquema de modelos de mezcladores 
Tabla 6: Dimensiones de mezcladores

\begin{tabular}{|c|c|c|c|c|c|}
\hline Modelo & $\begin{array}{l}\text { Long. Sección } \\
\text { convergente }(\mathrm{mm})\end{array}$ & $\begin{array}{l}\text { Ángulo de } \\
\left.\text { entrada ( }{ }^{\circ}\right)\end{array}$ & $\begin{array}{l}\text { Diametro de } \\
\text { garganta }(\mathrm{mm})\end{array}$ & $\begin{array}{l}\text { Long. Sección } \\
\text { divergente }(\mathrm{mm})\end{array}$ & $\begin{array}{l}\text { Ángulo de } \\
\left.\text { salida ( }{ }^{\circ}\right)\end{array}$ \\
\hline Representación & "a" & "a" & "d" & "b" & $" \beta "$ \\
\hline GLP-1 & 11,5 & 40 & 4.6 & 55,4 & 7 \\
\hline GLP-2 & 11,5 & 40 & 4.6 & 41.4 & 7 \\
\hline GLP-3 & 23 & 40 & 4.6 & 55,4 & 7 \\
\hline GLP-4 & 21.3 & 40 & 8.5 & 102.4 & 7 \\
\hline GLP-5 & 50 & 13.8 & 14 & - & - \\
\hline GLP-6 & 23 & 13.8 & 4.6 & 55,4 & 7 \\
\hline
\end{tabular}

\section{DISEÑO EXPERIMETAL}

Los resultados obtenidos presentan un cierto nivel de variabilidad, generada por la presencia de diversos factores, tales como: las incertidumbres establecidas por los equipos de medición o las probables fallas en las mediciones debido al operador.

En lo que respecta a la incertidumbre de lectura, esta se toma como la mitad de la división más pequeña de la escala de medición del elemento, tomando en cuenta que debe coincidir con las cifras significativas del instrumento usado para la medición (Thompson et al., 2003). En la Tabla 7 se muestran los valores de incertidumbre de lectura estimados para los equipos de medición.

Tabla 7: Incertidumbre de lectura de equipos

\begin{tabular}{|c|c|c|}
\hline Instrumento de medición & Incertidumbre & Unidades \\
\hline Caudalímetro & $\pm 0,01$ & l/min \\
\hline Termocupla & $\pm 0,01$ & o C \\
\hline Manómetro digital & $\pm 0,01$ & $\mathrm{mbar}$ \\
\hline Temperatura ambiental & $\pm 0,1$ & o C \\
\hline Humedad Relativa & $\pm 0,1$ & $\%$ \\
\hline Masa del agua & $\pm 0,001$ & $\mathrm{~kg}$ \\
\hline Cronómetro & $\pm 0,01$ & $\mathrm{~s}$ \\
\hline Analizador de gases (CO) & \pm 20 & $\mathrm{ppm}$ \\
\hline
\end{tabular}

Para el cálculo de la incertidumbre de lectura en el rendimiento térmico $\left(\sigma_{L}\right)$, desarrollada a partir de las incertidumbres de lectura de los instrumentos descritos en la Tabla 7, en la toma de datos de los ensayos, se desarrolló la ecuación 15 (Thompson et al. 2003):

$\sigma_{L}=\left[\left(\frac{\Delta m_{\text {agua }}}{m_{\text {agua }}}\right)+\left(\frac{2 \Delta T}{\left(T_{f}-T_{i}\right)}\right)+\left(\frac{\Delta t_{a}}{t_{a}}+\frac{\Delta \dot{Q}_{g}}{\dot{Q}_{g}}\right)\right] \cdot n$

Donde las variables utilizadas son las siguientes:

$\Delta \mathrm{m}_{\text {agua }}=$ Incertidumbre de lectura de la balanza electrónica usada para la medición de masa de agua $(\mathrm{kg})$

$\mathrm{m}_{\text {agua }}=$ Masa de agua $(\mathrm{kg})$

$\Delta \mathrm{T} \quad=$ Incertidumbre de lectura de la termocupla usada para la medición de temperatura del agua $\left({ }^{\circ} \mathrm{C}\right)$

$\mathrm{T}_{\mathrm{f}} \quad=$ Temperatura final del agua $\left({ }^{\circ} \mathrm{C}\right)$

$\mathrm{T}_{\mathrm{i}} \quad=$ Temperatura inicial del agua $\left({ }^{\circ} \mathrm{C}\right)$

$\Delta \mathrm{t}_{\mathrm{a}} \quad=$ Incertidumbre de lectura del cronómetro utilizado para medir el tiempo requerido para calentar el agua desde la temperatura $T_{i}$ hasta $T_{f}(\mathrm{~s})$

$\mathrm{t}_{\mathrm{a}} \quad=$ Tiempo requerido para calentar el agua desde la temperatura $T_{i}$ hasta $T_{f}(\mathrm{~s})$

$\Delta \dot{Q}_{\mathrm{g}} \quad=$ Incertidumbre del caudalímetro usado para medir el flujo del combustible utilizado $\left(\mathrm{m}^{3} / \mathrm{s}\right)$

$\Delta \dot{\mathrm{Q}}_{\mathrm{g}} \quad=$ Incertidumbre del flujo del combustible utilizado $\left(\mathrm{m}^{3} / \mathrm{s}\right)$

$\mathrm{n} \quad=$ Rendimiento térmico alcanzado (\%) 
Por otra parte, el control de los ensayos está sujeto a los sentidos del operador; por lo tanto, existe la posibilidad de error en la lectura de los instrumentos de medición o de mal posicionamiento de los elementos de trabajo, tales como la olla o el mezclador (Bisso, 2013). El conjunto de estas posibles fuentes de oscilaciones en los resultados se denominará como la incertidumbre debida a fluctuaciones $\left(\sigma_{F}\right)$. Es así que se realizaron tres ensayos para cada punto analizado, de modo que se tenga una mayor certeza en los valores obtenidos, siendo $\bar{x}$ la media aritmética de las medidas $x_{1}, x_{2}$ y $x_{3}$.

$\bar{x}=\frac{x_{1}+x_{2}+x_{3}}{3}$

Posteriormente, la incertidumbre debida a las fluctuaciones $\left(\sigma_{F}\right)$, obtenida tras los resultados de "N" ensayos, se calcula de la siguiente manera:

$\sigma_{\mathrm{F}}=\sqrt{\frac{\left(\mathrm{x}_{1}-\overline{\mathrm{x}}\right)^{2}+\left(\mathrm{x}_{2}-\overline{\mathrm{x}}\right)^{2}+\left(\mathrm{x}_{3}-\overline{\mathrm{x}}\right)^{2}}{\mathrm{~N} \cdot(\mathrm{N}-1)}}$

Finalmente, la incertidumbre total $\left(\sigma_{T}\right)$, la cual representa la combinación de la incertidumbre de lectura y de fluctuaciones, y cuyo valor se muestra desde la Tabla 8 hasta la 19, se estima de la siguiente forma:

$\sigma_{T}=\sqrt{\sigma_{L}{ }^{2}+\sigma_{F}{ }^{2}}$

\section{RESULTADOS Y DISCUSIÓN}

Como se mencionó anteriormente, las pruebas seguirán lo trazado por la norma NCh927/1. Debe mencionarse que se utilizó un tiempo prudencial entre cada ensayo, de modo que exista tiempo suficiente para enfriar las superficies del recipiente, manteniendo las mismas condiciones para todos los casos. Es así que se variarán las distancias entre la superficie inferior de la olla y el quemador, así como del mezclador y la salida del inyector de gas combustible; de forma que se pueda obtener las condiciones que posibiliten un mayor aprovechamiento de la energía liberada durante el proceso de combustión del gas.

Se iniciará con los ensayos que permitan estudiar la influencia que tiene la distancia entre el inyector y la entrada del mezclador en la calidad de la combustión en cada uno de los modelos propuestos. Considerando que el punto " 0,0 " es cuando la salida del inyector está al mismo nivel que la entrada del mezclador; luego, se modificará la distancia en tramos de $0,5 \mathrm{~cm}$, manteniendo siempre una distancia de 1 $\mathrm{cm}$ entre la superficie inferior de la olla y la hornilla del quemador. El objetivo de este proceso es localizar, de manera experimental, la distancia inyector-mezclador que posibilite un mayor aprovechamiento de la energía liberada durante el proceso de combustión del gas en el quemador. Asimismo, en cada punto, se procederá con los respectivos ensayos para calcular la cantidad de emisión de monóxido de carbono neutro provocada (Figura 5).

Cabe recalcar que, en algunos casos, no se pudo realizar pruebas con el inyector dentro del mezclador $(\mathrm{d}<0 \mathrm{~cm})$, debido a que las formas geométricas de los elementos impedían la inserción del inyector en la entrada del mezclador $y$, en otros casos, por el tamaño de la llama desarrollada en el quemador que imposibilitaba la ejecución de los respectivos ensayos. Finalmente, tras reconocer la longitud inyectormezclador que permite obtener un rendimiento térmico más elevado, se procederá a incrementar la altura de la superficie inferior de la olla con respecto a la hornilla del quemador, analizando el impacto que esto puede ocasionar en lo que se refiere a rendimiento térmico y emisión de monóxido de carbono neutro. Se realizarán tres ensayos de rendimiento térmico en cada punto a analizar, de manera que pueda demostrarse, con seguridad, que el porcentaje obtenido es el correcto, permitiendo a su vez el desarrollo del análisis de incertidumbre correspondiente.

\section{Modelo GLP-1}

En la Tabla 8 y en la Figura 6 se puede ver que, manteniendo una altura de $1 \mathrm{~cm}$ entre la olla con respecto a la hornilla $(\mathrm{h}=1 \mathrm{~cm})$ y una distancia entre el inyector y el mezclador de $0 \mathrm{~cm}(\mathrm{~d}=0,0 \mathrm{~cm})$, se obtiene el nivel más alto de rendimiento térmico; sin embargo, no supera el límite estipulado por la norma (58\%). Además, los niveles de porcentaje de emisión de $\mathrm{CO}_{\mathrm{n}}$ están por encima de lo máximo admisible. 


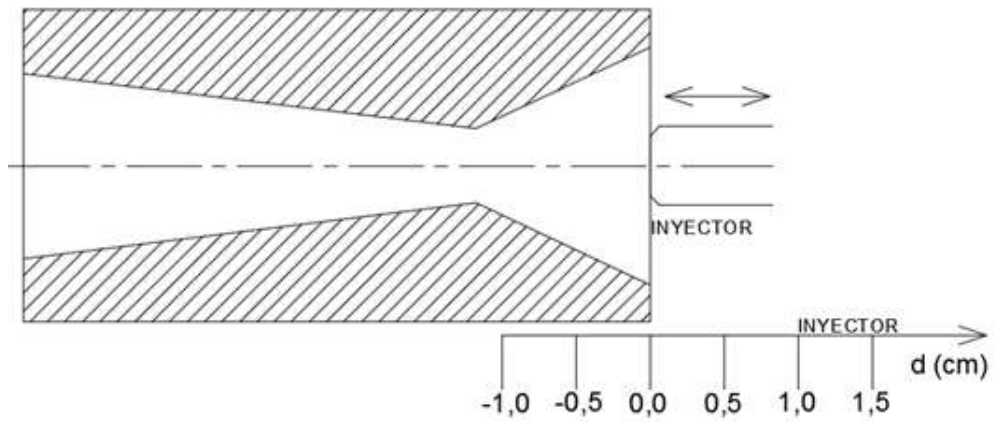

Fig. 5: Representación de variación en la distancia "d" entre el inyector y el mezclador

Tabla 8: Rendimiento térmico y porcentaje de monóxido de carbono neutro usando el mezclador GLP-1, según la distancia "d" del inyector con respecto al mezclador

\begin{tabular}{|c|c|c|c|c|}
\hline $\begin{array}{l}\text { Distancia "d" } \\
(\mathrm{cm})\end{array}$ & $\begin{array}{c}\text { Mediciones de } \\
\text { Rendimiento Térmico (\%) }\end{array}$ & $\begin{array}{c}\text { Rendimiento Térmico } \\
\text { Promedio (\%) }\end{array}$ & $\begin{array}{c}\text { Incertidumbre Total } \\
(\%)\end{array}$ & $\begin{array}{c}\text { Monóxido de carbono } \\
\text { neutro (\%) }\end{array}$ \\
\hline \multirow{3}{*}{0,0} & 55,24 & \multirow{3}{*}{55,62} & \multirow{3}{*}{0,59} & \multirow{3}{*}{0,6578} \\
\hline & 55,97 & & & \\
\hline & 55,66 & & & \\
\hline \multirow{3}{*}{0,5} & 55,38 & \multirow{3}{*}{55,33} & \multirow{3}{*}{0,60} & \multirow{3}{*}{0,6012} \\
\hline & 54,88 & & & \\
\hline & 55,75 & & & \\
\hline \multirow{3}{*}{1,0} & 55,15 & \multirow{3}{*}{55,09} & \multirow{3}{*}{0,69} & \multirow{3}{*}{0,5226} \\
\hline & 54,31 & & & \\
\hline & 55,80 & & & \\
\hline \multirow{3}{*}{1,5} & 54,60 & \multirow{3}{*}{54,72} & \multirow{3}{*}{0,64} & \multirow{3}{*}{0,4551} \\
\hline & 54,18 & & & \\
\hline & 55,40 & & & \\
\hline \multirow{3}{*}{2,0} & 53.97 & \multirow{3}{*}{53.83} & \multirow{3}{*}{0,73} & \multirow{3}{*}{0,4054} \\
\hline & 54,60 & & & \\
\hline & 52.93 & & & \\
\hline
\end{tabular}

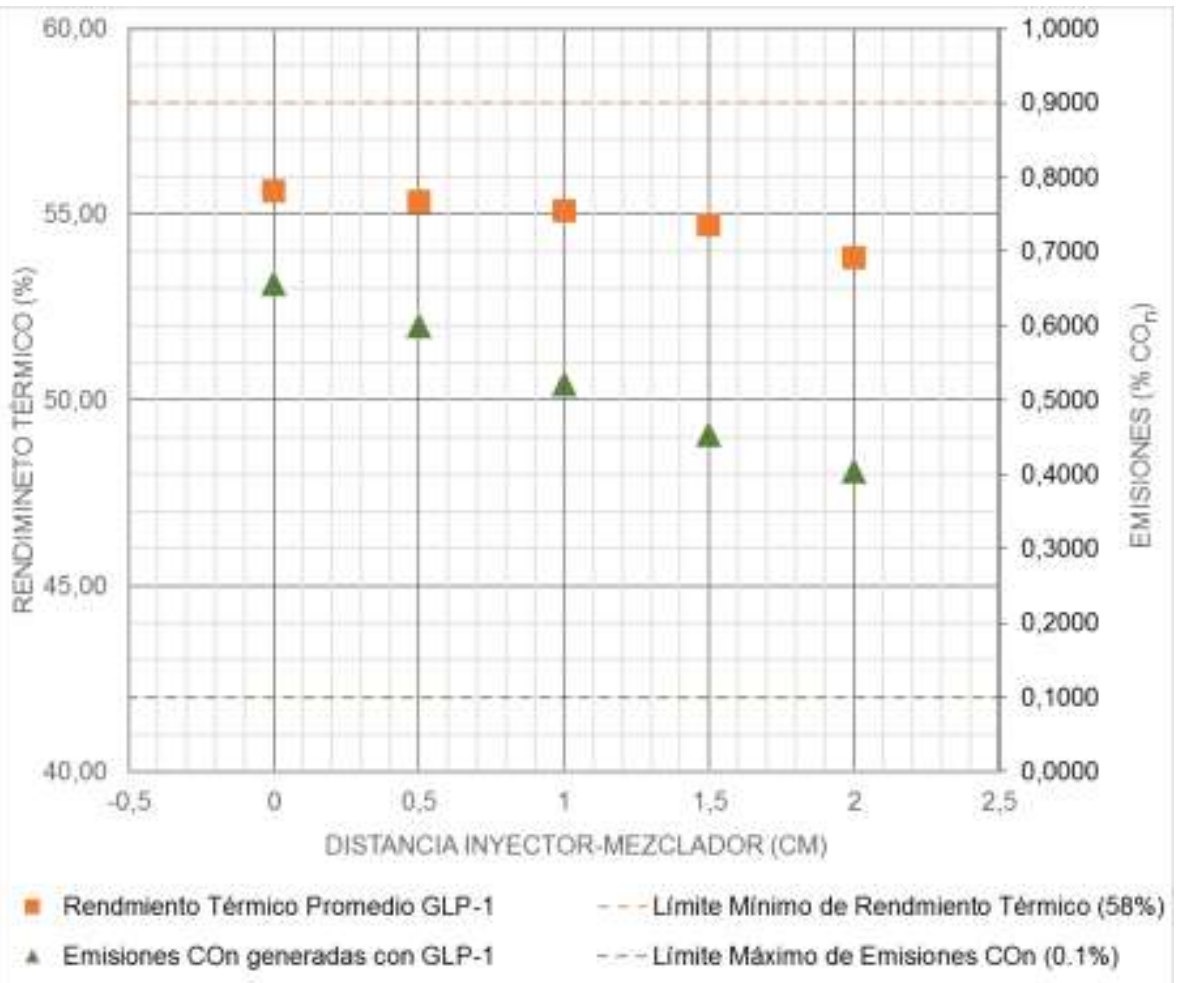

Fig. 6: Rendimiento térmico y emisiones de $\mathrm{CO}_{n}$ influenciados por distancia "d" mezcladorinyector en mezclador GLP-1 
En la Tabla 9 y Figura 7 se puede ver que al elevar la distancia entre la olla y la hornilla hasta $3 \mathrm{~cm}(\mathrm{~h}=3 \mathrm{~cm})$ y manteniendo una distancia entre el inyector y el mezclador de $0,0 \mathrm{~cm}(\mathrm{~d}=0,0 \mathrm{~cm})$ el porcentaje de monóxido de carbono neutro se reduce hasta estar cerca de lo permisible, aunque el rendimiento se reduce hasta llegar a un nivel por debajo de lo permisible.

Tabla 9: Rendimiento térmico y porcentaje de monóxido de carbono neutro usando el mezclador GLP-1, según la altura "h" entre la superficie inferior de la olla y la hornilla, manteniendo una distancia de $0,0 \mathrm{~cm}$ entre el inyector y el mezclador

\begin{tabular}{|c|c|c|c|c|}
\hline Altura "h" (cm) & \begin{tabular}{|l} 
Mediciones de \\
Rendimiento Térmico (\%)
\end{tabular} & $\begin{array}{l}\text { Rendimiento Térmico } \\
\text { Promedio (\%) }\end{array}$ & $\begin{array}{l}\text { Incertidumbre Total } \\
\text { (\%) }\end{array}$ & $\begin{array}{l}\text { Monóxido de carbono } \\
\text { neutro (\%) }\end{array}$ \\
\hline \multirow{3}{*}{1,0} & 55,24 & \multirow{3}{*}{55,62} & \multirow{3}{*}{0,59} & \multirow{3}{*}{0,6578} \\
\hline & 55,97 & & & \\
\hline & 55,66 & & & \\
\hline \multirow{3}{*}{1,5} & 55,15 & \multirow{3}{*}{55,40} & \multirow{3}{*}{0,69} & \multirow{3}{*}{0,4868} \\
\hline & 56,21 & & & \\
\hline & 54,85 & & & \\
\hline \multirow{3}{*}{2,0} & 55,29 & \multirow{3}{*}{55,23} & \multirow{3}{*}{0,59} & \multirow{3}{*}{0,3972} \\
\hline & 54,81 & & & \\
\hline & 55,59 & & & \\
\hline \multirow{3}{*}{2,5} & 54,50 & \multirow{3}{*}{54,44} & \multirow{3}{*}{0,56} & \multirow{3}{*}{0,2896} \\
\hline & 54,65 & & & \\
\hline & 54,17 & & & \\
\hline \multirow{3}{*}{3,0} & 53.55 & \multirow{3}{*}{53.94} & \multirow{3}{*}{0,58} & \multirow{3}{*}{0,1338} \\
\hline & 53.96 & & & \\
\hline & 54,31 & & & \\
\hline
\end{tabular}

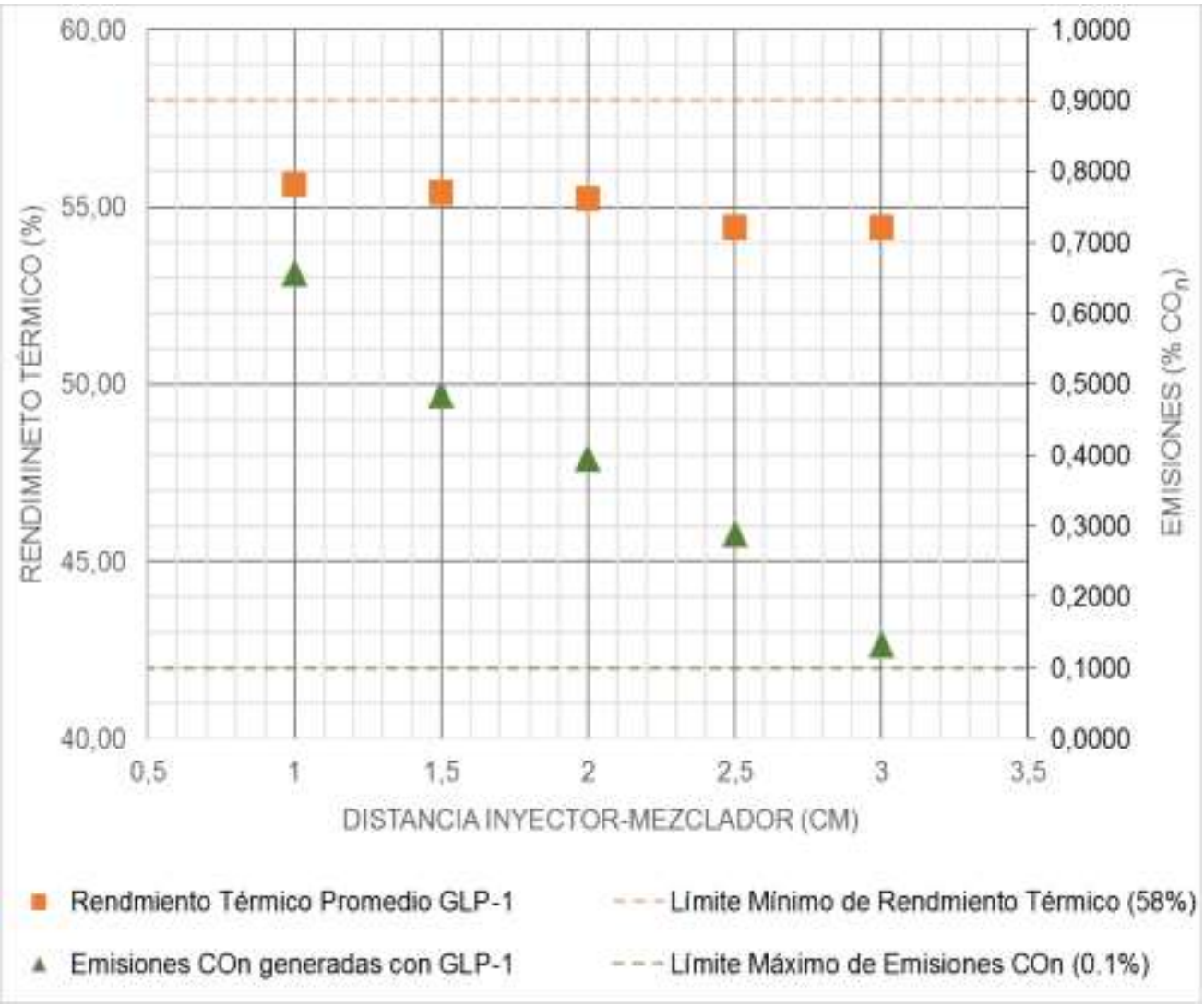

Fig. 7: Rendimiento térmico y emisiones de $\mathrm{CO}_{n}$ influenciados por la altura "h" quemadorolla en mezclador GLP -1 


\section{Modelo GLP-2}

Según los resultados mostrados en la Tabla 10 y Figura 8, manteniendo una altura de $1 \mathrm{~cm}$ entre la olla con respecto a la hornilla $(h=1 \mathrm{~cm})$ y una distancia entre el inyector y el mezclador de $0,5 \mathrm{~cm}(\mathrm{~d}=0,5 \mathrm{~cm})$, se obtiene el mayor nivel de rendimiento térmico, aunque sin superar el límite de rendimiento térmico admisible $(58,0 \%)$, aunque si se considera la incertidumbre no puede asegurarse que su rendimiento siempre estará por debajo de $58,0 \%$ a estas condiciones (ver Tabla 10). Por su parte, las emisiones de $\mathrm{CO}_{\mathrm{n}}$ están por encima lo máximo recomendado.

Tabla 10: Rendimiento térmico y porcentaje de monóxido de carbono neutro usando el mezclador GLP-2, según la distancia "d" del inyector con respecto al mezclador

\begin{tabular}{|c|c|c|c|c|}
\hline $\begin{array}{l}\text { Distancia "d" } \\
(\mathrm{cm})\end{array}$ & $\begin{array}{c}\text { Mediciones de } \\
\text { Rendimiento Térmico (\%) }\end{array}$ & $\begin{array}{c}\text { Rendimiento Térmico } \\
\text { Promedio (\%) }\end{array}$ & $\begin{array}{c}\text { Incertidumbre Total } \\
(\%)\end{array}$ & $\begin{array}{c}\text { Monóxido de carbono } \\
\text { neutro (\%) }\end{array}$ \\
\hline \multirow{3}{*}{0,0} & 56,09 & \multirow{3}{*}{55,86} & \multirow{3}{*}{0,72} & \multirow{3}{*}{0,6812} \\
\hline & 56,53 & & & \\
\hline & 54,97 & & & \\
\hline \multirow{3}{*}{0,5} & 57,76 & \multirow{3}{*}{57,36} & \multirow{3}{*}{0,72} & \multirow{3}{*}{0,5999} \\
\hline & 57,84 & & & \\
\hline & 56,47 & & & \\
\hline \multirow{3}{*}{1,0} & 56,88 & \multirow{3}{*}{56,70} & \multirow{3}{*}{0,57} & \multirow{3}{*}{0,5378} \\
\hline & 56,77 & & & \\
\hline & 56,46 & & & \\
\hline \multirow{3}{*}{1,5} & 54,28 & \multirow{3}{*}{54,27} & \multirow{3}{*}{0,68} & \multirow{3}{*}{0,5144} \\
\hline & 54,99 & & & \\
\hline & 53.55 & & & \\
\hline \multirow{3}{*}{2,0} & 53.27 & \multirow{3}{*}{53.35} & \multirow{3}{*}{0,53} & \multirow{3}{*}{0,4413} \\
\hline & 53.23 & & & \\
\hline & 53.54 & & & \\
\hline
\end{tabular}

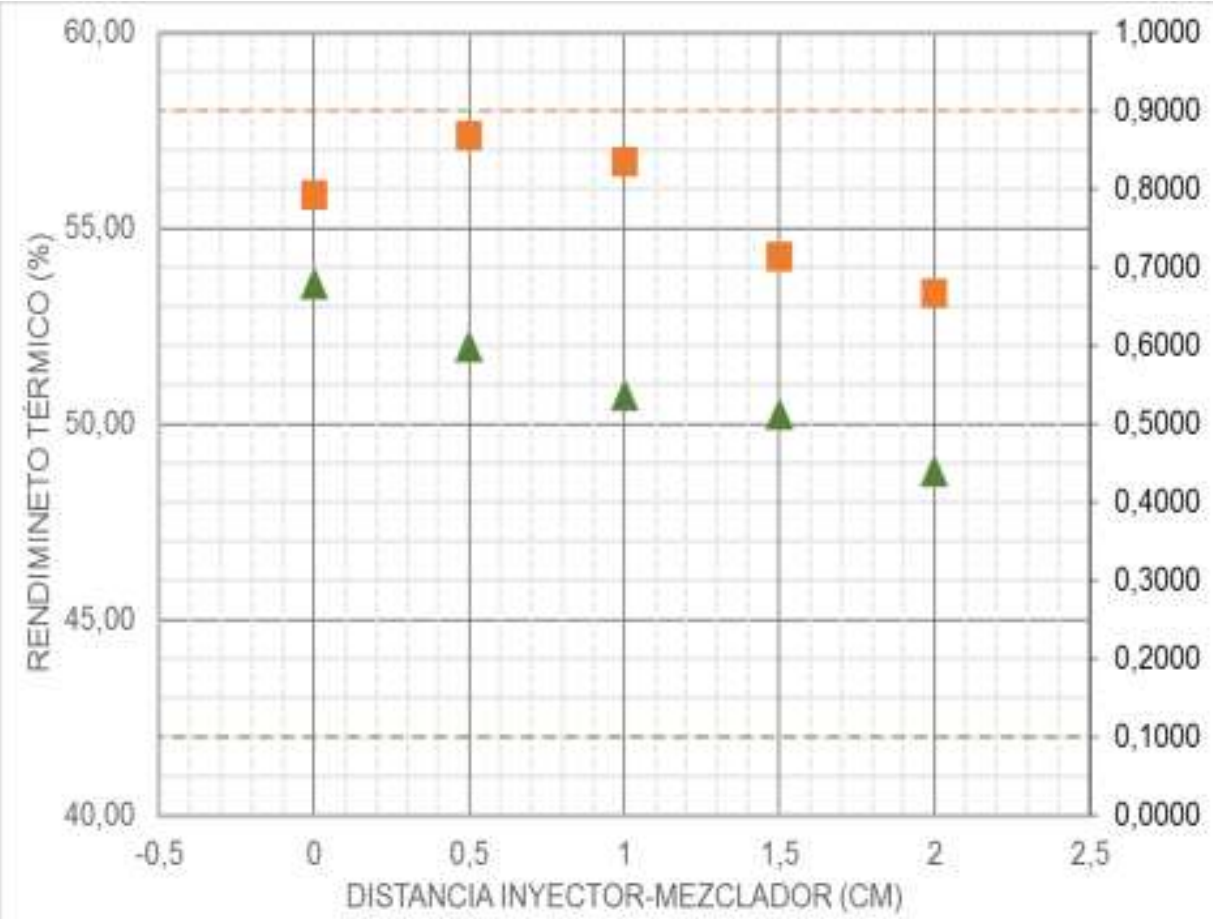

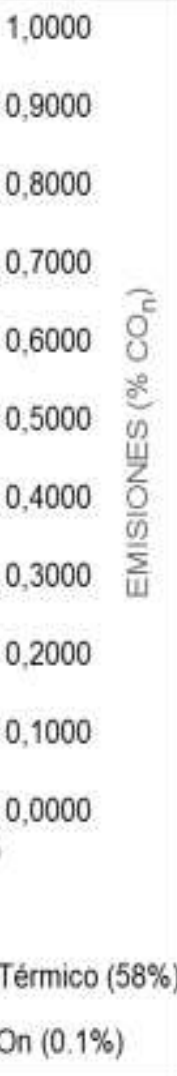

Fig. 8: Rendimiento térmico y emisiones de $\mathrm{CO}_{\mathrm{n}}$ influenciados por distancia "d" mezcladorinyector en el mezclador GLP-2 
Como se puede ver en la Tabla 11 y Figura 9, al igual que en el mezclador GLP-1, en este modelo las emisiones de Monóxido de Carbono Neutro se reducen a medida que se eleva la distancia entre el quemador y superficie inferior de la olla; sin embargo, no se obtiene un valor cercano al valor límite de $\mathrm{CO}_{\mathrm{n}}$ permitido por la norma.

Tabla 11: Rendimiento térmico y porcentaje de monóxido de carbono neutro usando el mezclador GLP-2, según la altura "h" entre la superficie inferior de la olla y la hornilla; manteniendo una distancia de $0,5 \mathrm{~cm}$ entre el inyector y el mezclador

\begin{tabular}{|c|c|c|c|c|}
\hline Altura "h" (cm) & $\begin{array}{l}\text { Mediciones de } \\
\text { Rendimiento Térmico (\%) }\end{array}$ & $\begin{array}{l}\text { Rendimiento Térmico } \\
\text { Promedio (\%) }\end{array}$ & $\begin{array}{l}\text { Incertidumbre Total } \\
(\%)\end{array}$ & $\begin{array}{l}\text { Monóxido de carbono } \\
\text { neutro }(\%)\end{array}$ \\
\hline \multirow{3}{*}{1,0} & 57,76 & \multirow{3}{*}{57,36} & \multirow{3}{*}{0,72} & \multirow{3}{*}{0,5999} \\
\hline & 57,84 & & & \\
\hline & 56,47 & & & \\
\hline \multirow{3}{*}{1,5} & 57,84 & \multirow{3}{*}{57,60} & \multirow{3}{*}{0,62} & \multirow{3}{*}{0,4371} \\
\hline & 57,14 & & & \\
\hline & 57,81 & & & \\
\hline \multirow{3}{*}{2,0} & 56,58 & \multirow{3}{*}{56,49} & \multirow{3}{*}{0,56} & \multirow{3}{*}{0,3461} \\
\hline & 56,40 & & & \\
\hline & 56,50 & & & \\
\hline \multirow{3}{*}{2,5} & 53.75 & \multirow{3}{*}{54,40} & \multirow{3}{*}{0,66} & \multirow{3}{*}{0,2703} \\
\hline & 55,05 & & & \\
\hline & 54,39 & & & \\
\hline \multirow{3}{*}{3,0} & 51.24 & \multirow{3}{*}{52.40} & \multirow{3}{*}{0,78} & \multirow{3}{*}{0,2151} \\
\hline & 53.12 & & & \\
\hline & 52.83 & & & \\
\hline
\end{tabular}

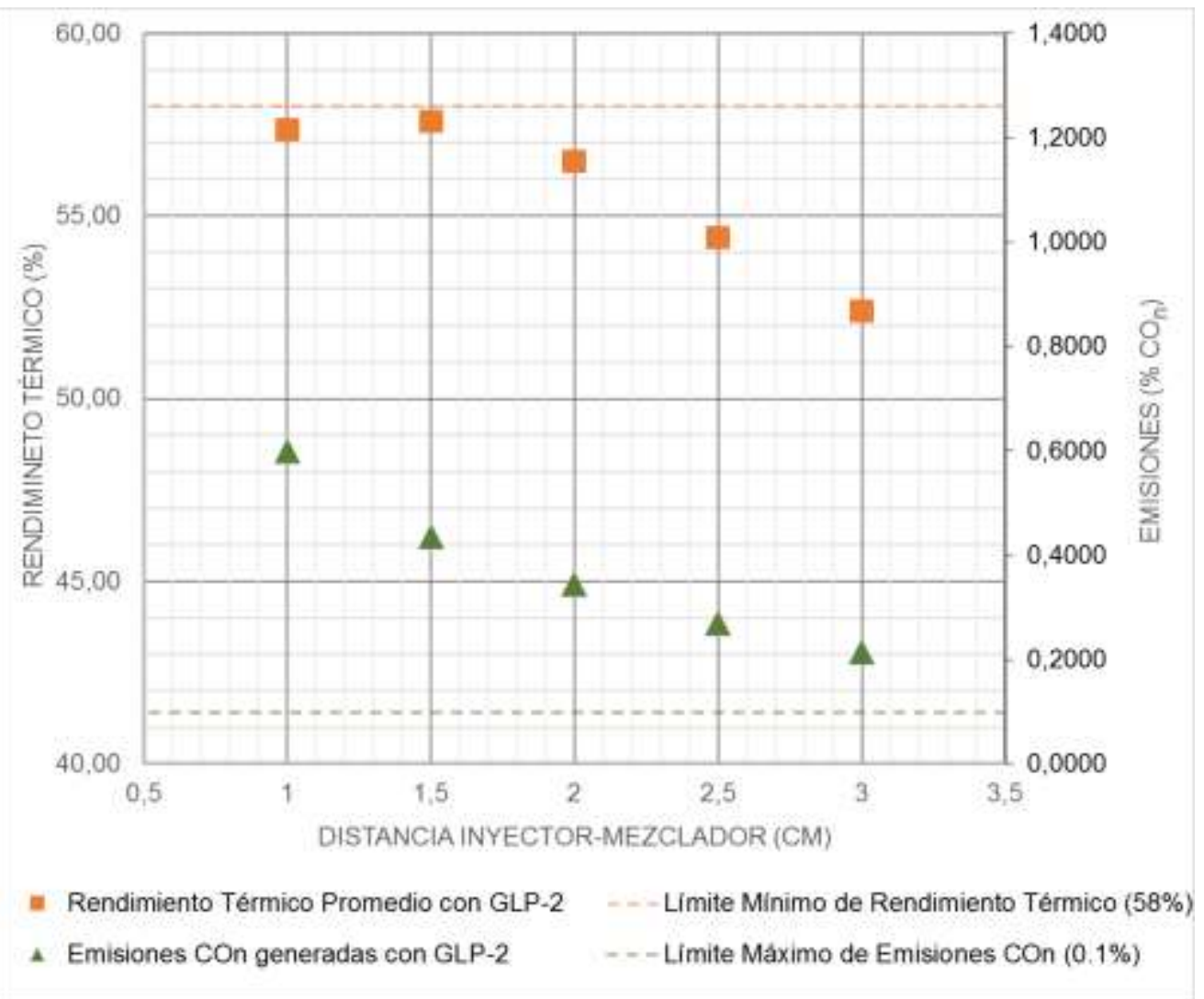

Fig. 9: Gráfico de Rendimiento y emisiones de $\mathrm{CO}_{\mathrm{n}}$ influenciados por la altura "h" quemadorolla en el mezclador GLP-2 


\section{Modelo GLP-3}

En el mezclador GLP-3, siguiendo lo mostrado en la Tabla 12 y en la Figura 10, se obtuvo el punto de rendimiento térmico más alto cuando la distancia entre el inyector y el mezclador fue de $0,0 \mathrm{~cm}(\mathrm{~d}=0,0 \mathrm{~cm})$ y manteniendo una altura entre la hornilla y la olla de $1 \mathrm{~cm}(\mathrm{~h}=1,0 \mathrm{~cm})$; sin embargo, no supera el límite mínimo requerido, ni lo obtenido con los mezcladores GLP-1 y GLP-2. A su vez, se muestran niveles de emisiones de $\mathrm{CO}_{\mathrm{n}}$ por encima del límite máximo.

Tabla 12: Rendimiento térmico y porcentaje de monóxido de carbono neutro usando el mezclador GLP-3, según la distancia "d" del inyector con respecto al mezclador

\begin{tabular}{|c|c|c|c|c|}
\hline $\begin{array}{l}\text { Distancia "d" } \\
(\mathrm{cm})\end{array}$ & $\begin{array}{c}\text { Mediciones de } \\
\text { Rendimiento Térmico (\%) }\end{array}$ & $\begin{array}{c}\text { Rendimiento Térmico } \\
\text { Promedio (\%) }\end{array}$ & \begin{tabular}{|c|} 
Incertidumbre Total \\
$(\%)$
\end{tabular} & $\begin{array}{c}\text { Monóxido de carbono } \\
\text { neutro (\%) }\end{array}$ \\
\hline \multirow{3}{*}{$-0,5$} & 54,13 & \multirow{3}{*}{54,88} & \multirow{3}{*}{0,68} & \multirow{3}{*}{0,4661} \\
\hline & 54,93 & & & \\
\hline & 55,57 & & & \\
\hline \multirow{3}{*}{0,0} & 55,14 & \multirow{3}{*}{55,63} & \multirow{3}{*}{0,68} & \multirow{3}{*}{0,4151} \\
\hline & 56,43 & & & \\
\hline & 55,32 & & & \\
\hline \multirow{3}{*}{0,5} & 55,27 & \multirow{3}{*}{55,14} & \multirow{3}{*}{0,64} & \multirow{3}{*}{0,3723} \\
\hline & 54,49 & & & \\
\hline & 55,66 & & & \\
\hline \multirow{3}{*}{1,0} & 54,35 & \multirow{3}{*}{54,58} & \multirow{3}{*}{0,72} & \multirow{3}{*}{0,2565} \\
\hline & 53.87 & & & \\
\hline & 55,51 & & & \\
\hline \multirow{3}{*}{1,5} & 52.46 & \multirow{3}{*}{51.74} & \multirow{3}{*}{1,09} & \multirow{3}{*}{0,2137} \\
\hline & 52.93 & & & \\
\hline & 49.83 & & & \\
\hline
\end{tabular}

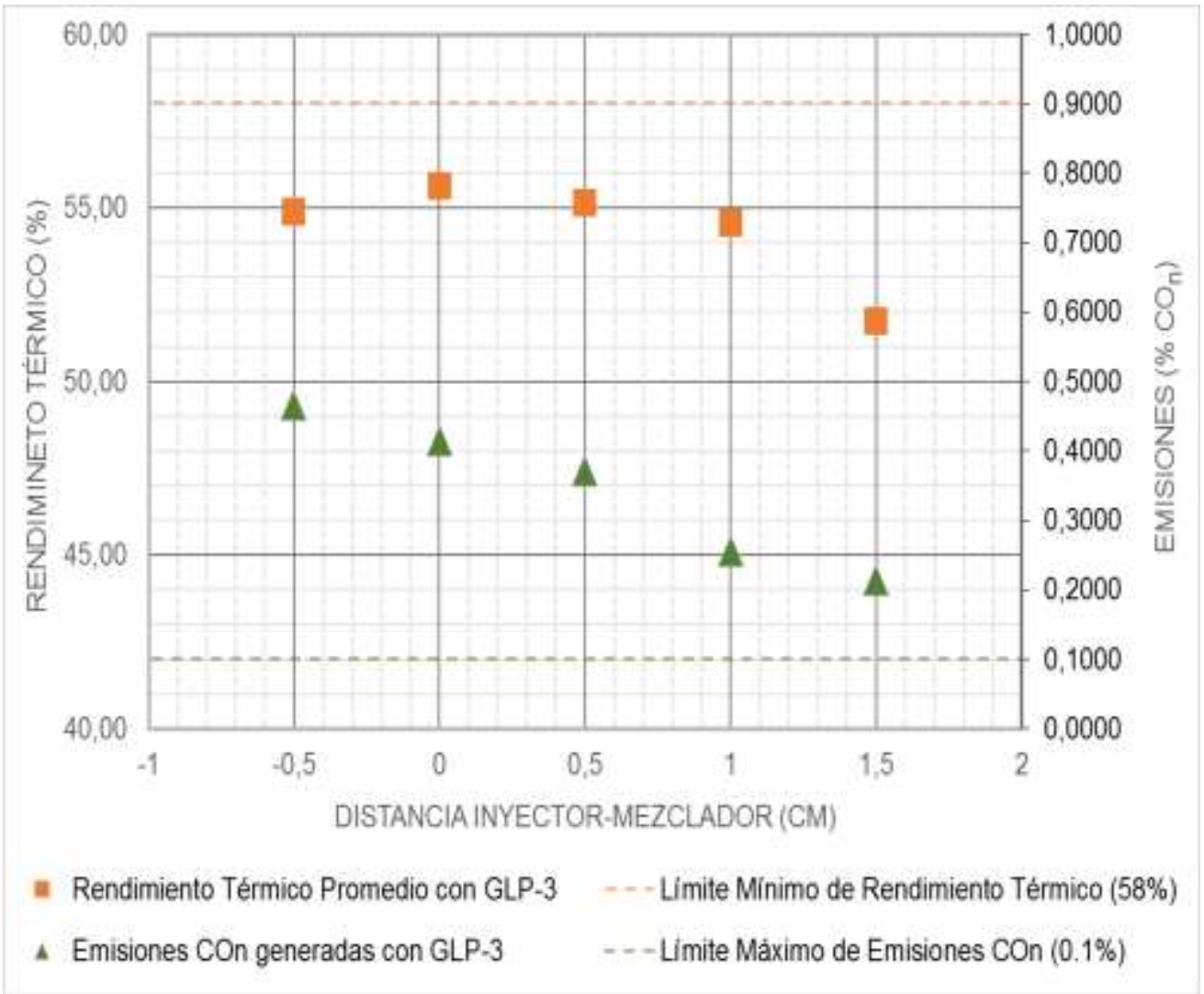

Fig. 10: Rendimiento térmico y emisiones de $\mathrm{CO}_{\mathrm{n}}$ influenciados por la distancia "d" mezclador-inyector en el mezclador GLP-3 
En la Tabla 13 y en la Figura 11 se puede observar que al alejar la olla de la hornilla el rendimiento térmico no muestra alguna considerable variación; sin embargo, las emisiones de monóxido de carbono neutro presentan una reducción, en promedio de $15 \%$ cada vez que se aleja 0,5 centímetros la olla del quemador.

Tabla 13: Rendimiento térmico y porcentaje de monóxido de carbono neutro usando el mezclador GLP-3, según la altura "h" entre la superficie inferior de la olla y la hornilla; manteniendo una distancia de 0,0 cm entre el inyector y el mezclador

\begin{tabular}{|c|c|c|c|c|}
\hline Altura " $h "$ (cm) & $\begin{array}{c}\text { Mediciones de } \\
\text { Rendimiento Térmico (\%) }\end{array}$ & $\begin{array}{l}\text { Rendimiento Térmico } \\
\text { Promedio (\%) }\end{array}$ & $\begin{array}{c}\text { Incertidumbre Total } \\
(\%)\end{array}$ & $\begin{array}{c}\text { Monóxido de carbono } \\
\text { neutro (\%) }\end{array}$ \\
\hline \multirow{3}{*}{1,0} & 55,14 & \multirow{3}{*}{55,63} & \multirow{3}{*}{0,69} & \multirow{3}{*}{0,4151} \\
\hline & 56,43 & & & \\
\hline & 55,32 & & & \\
\hline \multirow{3}{*}{1,5} & 55,15 & \multirow{3}{*}{55,44} & \multirow{3}{*}{0,57} & \multirow{3}{*}{0,3310} \\
\hline & 55,75 & & & \\
\hline & 55,43 & & & \\
\hline \multirow{3}{*}{2,0} & 55,68 & \multirow{3}{*}{55,19} & \multirow{3}{*}{0,60} & \multirow{3}{*}{0,3103} \\
\hline & 55,04 & & & \\
\hline & 54,84 & & & \\
\hline \multirow{3}{*}{2,5} & 55,34 & \multirow{3}{*}{55,14} & \multirow{3}{*}{0,56} & \multirow{3}{*}{0,2524} \\
\hline & 55,03 & & & \\
\hline & 55,04 & & & \\
\hline \multirow{3}{*}{3,0} & 53.33 & \multirow{3}{*}{53.72} & \multirow{3}{*}{0,58} & \multirow{3}{*}{0,1365} \\
\hline & 54,07 & & & \\
\hline & 53.76 & & & \\
\hline
\end{tabular}

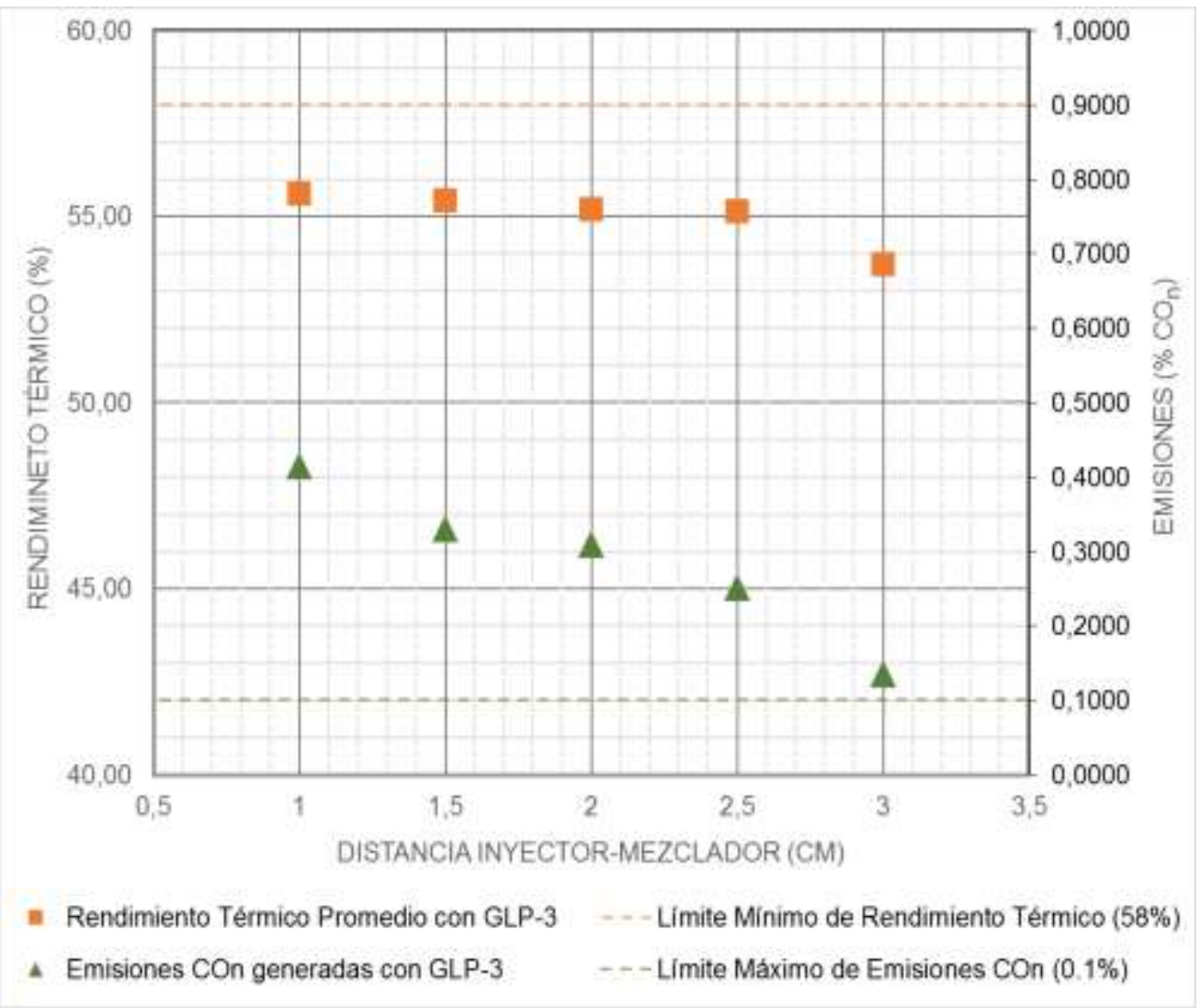

Fig. 11: Rendimiento térmico y emisiones de $\mathrm{CO}_{\mathrm{n}}$ influenciados por la altura "h" quemadorolla en el mezclador GLP-3 


\section{Modelo GLP-4}

El mezclador GLP-4, tal como se puede ver en la Tabla 14 y en la Figura 12, presenta el punto más alto de rendimiento térmico al establecerse una distancia "d" de 0,5 cm y una altura "h" de 1,0 cm entre la olla y la hornilla, aunque por debajo del límite requerido (58\%).

Tabla 14: Rendimiento térmico y porcentaje de monóxido de carbono neutro usando el mezclador GLP-4, según la distancia "d" del inyector con respecto al mezclador

\begin{tabular}{|c|c|c|c|c|}
\hline $\begin{array}{l}\text { Distancia "d" } \\
\text { (cm) }\end{array}$ & $\begin{array}{c}\text { Mediciones de } \\
\text { Rendimiento Térmico (\%) }\end{array}$ & $\begin{array}{l}\text { Rendimiento Térmico } \\
\text { Promedio (\%) }\end{array}$ & $\begin{array}{c}\text { Incertidumbre Total } \\
(\%)\end{array}$ & $\begin{array}{c}\text { Monóxido de carbono } \\
\text { neutro (\%) }\end{array}$ \\
\hline \multirow{3}{*}{$-0,5$} & 54,92 & \multirow{3}{*}{54,93} & \multirow{3}{*}{0,55} & \multirow{3}{*}{0,6909} \\
\hline & 54,77 & & & \\
\hline & 55,09 & & & \\
\hline \multirow{3}{*}{0} & 55,89 & \multirow{3}{*}{55,09} & \multirow{3}{*}{0,79} & \multirow{3}{*}{0,5881} \\
\hline & 54,01 & & & \\
\hline & 55,37 & & & \\
\hline \multirow{3}{*}{0,5} & 55,94 & \multirow{3}{*}{55,88} & \multirow{3}{*}{0,56} & \multirow{3}{*}{0,5592} \\
\hline & 55,71 & & & \\
\hline & 55,99 & & & \\
\hline \multirow{3}{*}{1} & 55,85 & \multirow{3}{*}{55,79} & \multirow{3}{*}{0,56} & \multirow{3}{*}{0,5337} \\
\hline & 55,95 & & & \\
\hline & 55,57 & & & \\
\hline \multirow{3}{*}{1,5} & 52.20 & \multirow{3}{*}{52.23} & \multirow{3}{*}{0,53} & \multirow{3}{*}{0,4744} \\
\hline & 52,08 & & & \\
\hline & 52.42 & & & \\
\hline
\end{tabular}

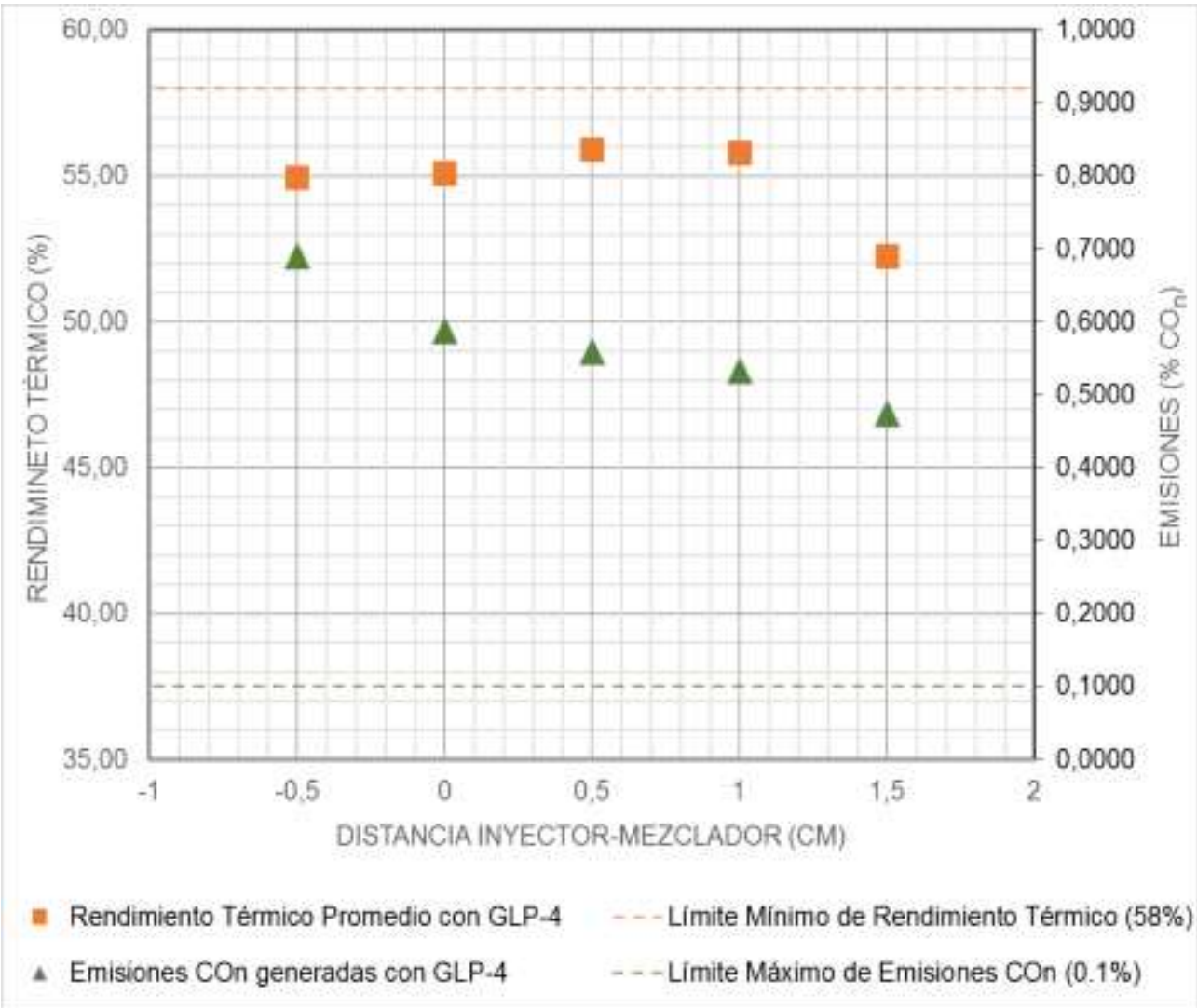

Fig. 12: Rendimiento térmico y emisiones de $\mathrm{CO}_{\mathrm{n}}$ influenciados por la distancia "d" mezclador-inyector en el mezclador GLP-4 
A su vez, si se mantiene una distancia "d" de $0,5 \mathrm{~cm}$ y se eleva la altura "h" a 3,0 cm, se puede observar en la Tabla 15 y en la Figura 13 que el porcentaje de emisiones de monóxido de carbono neutro supera aproximadamente al doble del máximo valor recomendado.

Tabla 15: Rendimiento térmico y porcentaje de monóxido de carbono neutro usando el mezclador GLP-4, según la altura "h" entre la superficie inferior de la olla y la hornilla, manteniendo una distancia de $0,5 \mathrm{~cm}$ entre el inyector y el mezclador

\begin{tabular}{|c|c|c|c|c|}
\hline Altura "h" (cm) & $\begin{array}{c}\text { Mediciones de } \\
\text { Rendimiento Térmico (\%) }\end{array}$ & $\begin{array}{c}\text { Rendimiento Térmico } \\
\text { Promedio (\%) }\end{array}$ & \begin{tabular}{|c|}
$\begin{array}{c}\text { Incertidumbre Total } \\
(\%)\end{array}$ \\
\end{tabular} & $\begin{array}{c}\text { Monóxido de carbono } \\
\text { neutro (\%) }\end{array}$ \\
\hline \multirow{3}{*}{1} & 55,94 & \multirow{3}{*}{55,88} & \multirow{3}{*}{0,56} & \multirow{3}{*}{0,5599} \\
\hline & 55,71 & & & \\
\hline & 55,99 & & & \\
\hline \multirow{3}{*}{1,5} & 55,67 & \multirow{3}{*}{55,62} & \multirow{3}{*}{0,57} & \multirow{3}{*}{0,5047} \\
\hline & 55,31 & & & \\
\hline & 55,87 & & & \\
\hline \multirow{3}{*}{2} & 55,15 & \multirow{3}{*}{55,23} & \multirow{3}{*}{0,56} & \multirow{3}{*}{0,4530} \\
\hline & 55,48 & & & \\
\hline & 55,04 & & & \\
\hline \multirow{3}{*}{2,5} & 54,60 & \multirow{3}{*}{54,93} & \multirow{3}{*}{0,58} & \multirow{3}{*}{0,3875} \\
\hline & 54,87 & & & \\
\hline & 55,32 & & & \\
\hline \multirow{3}{*}{3} & 54,03 & \multirow{3}{*}{54,05} & \multirow{3}{*}{0,55} & \multirow{3}{*}{0,2779} \\
\hline & 53.81 & & & \\
\hline & 54,33 & & & \\
\hline
\end{tabular}

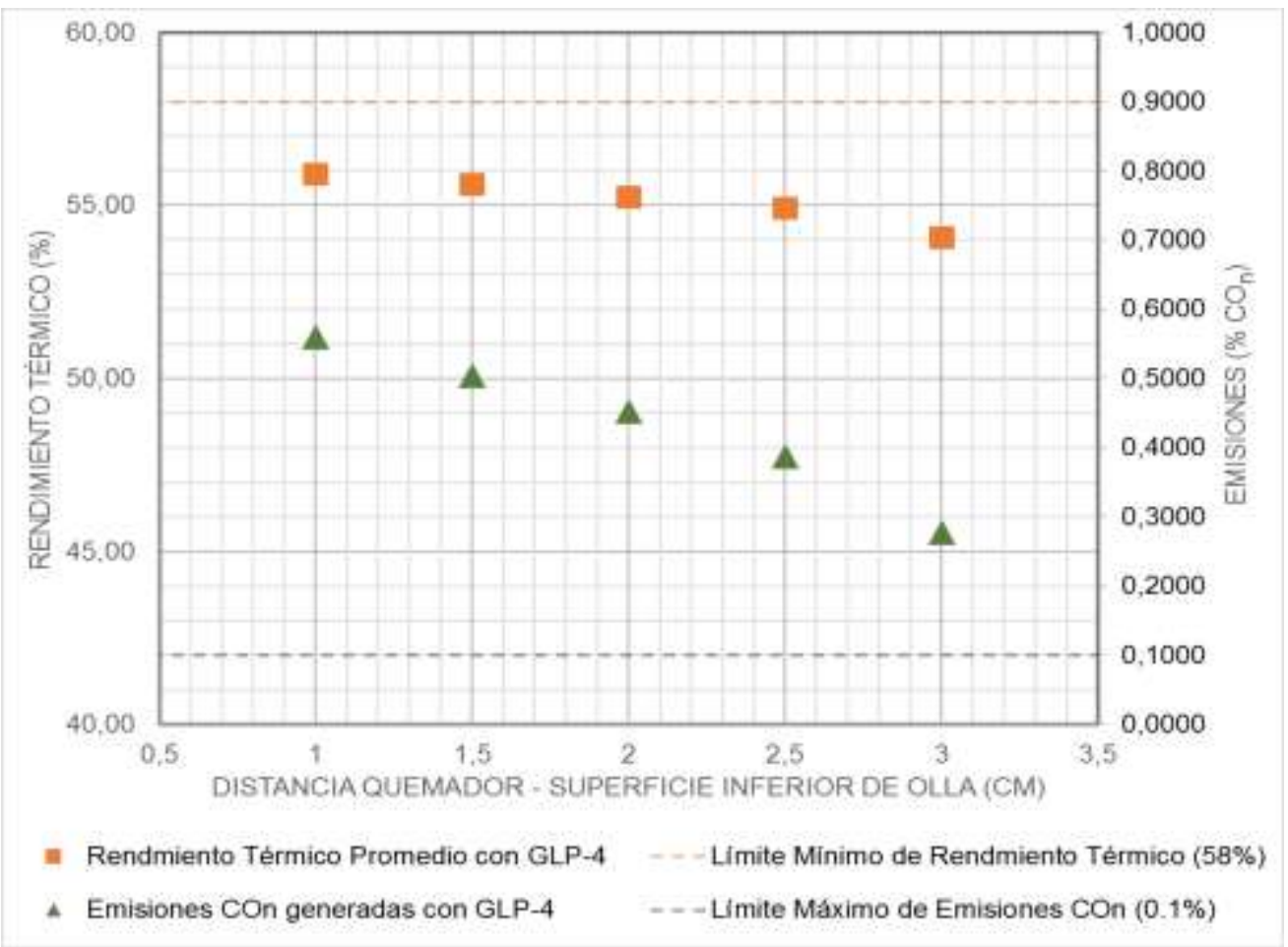

Fig. 13: Rendimiento térmico y emisiones de $\mathrm{CO}_{\mathrm{n}}$ influenciados por la altura "h" quemadorolla en mezclador GLP-4

\section{Modelo GLP-5}

Como se puede ver en la Tabla 16 y en la Figura 14, en el mezclador GLP-5 se obtiene el mayor nivel de rendimiento térmico cuando el inyector está introducido en el mezclador $0,5 \mathrm{~cm}(\mathrm{~d}=-0,5 \mathrm{~cm})$, superando el mínimo valor requerido $(58 \%)$ y lo obtenido en otros mezcladores. Sin embargo, se supera el límite máximo de porcentaje de emisiones de monóxido de carbono neutro $\left(\mathrm{CO}_{n}\right)$. 
Tabla 16: Rendimiento térmico y porcentaje de monóxido de carbono neutro usando el mezclador GLP-5, según la distancia "d" del inyector con respecto al mezclador

\begin{tabular}{|c|c|c|c|c|}
\hline $\begin{array}{l}\text { Distancia "d" } \\
(\mathrm{cm})\end{array}$ & $\begin{array}{c}\text { Mediciones de } \\
\text { Rendimiento Térmico (\%) }\end{array}$ & $\begin{array}{l}\text { Rendimiento Térmico } \\
\text { Promedio (\%) }\end{array}$ & $\begin{array}{c}\text { Incertidumbre Total } \\
(\%)\end{array}$ & $\begin{array}{c}\text { Monóxido de carbono } \\
\text { neutro (\%) }\end{array}$ \\
\hline \multirow{3}{*}{$-1,5$} & 57,27 & \multirow{3}{*}{57,38} & \multirow{3}{*}{0,58} & \multirow{3}{*}{0,7378} \\
\hline & 57,65 & & & \\
\hline & 57,23 & & & \\
\hline \multirow{3}{*}{$-1,0$} & 58,72 & \multirow{3}{*}{57,81} & \multirow{3}{*}{0,74} & \multirow{3}{*}{0,6674} \\
\hline & 57,40 & & & \\
\hline & 57,29 & & & \\
\hline \multirow{3}{*}{$-0,5$} & 58,49 & \multirow{3}{*}{58,25} & \multirow{3}{*}{0,90} & \multirow{3}{*}{0,5157} \\
\hline & 59.31 & & & \\
\hline & 56,95 & & & \\
\hline \multirow{3}{*}{0,0} & 57,35 & \multirow{3}{*}{58,37} & \multirow{3}{*}{0,78} & \multirow{3}{*}{0,5364} \\
\hline & 58,66 & & & \\
\hline & 59.12 & & & \\
\hline \multirow{3}{*}{0,5} & 56,21 & \multirow{3}{*}{56,77} & \multirow{3}{*}{0,63} & \multirow{3}{*}{0,4744} \\
\hline & 57,05 & & & \\
\hline & 57,06 & & & \\
\hline \multirow{3}{*}{1,0} & 54,02 & \multirow{3}{*}{53.80} & \multirow{3}{*}{0,75} & \multirow{3}{*}{0,4275} \\
\hline & 52.80 & & & \\
\hline & 54,56 & & & \\
\hline \multirow{3}{*}{1,5} & 47.66 & \multirow{3}{*}{47.88} & \multirow{3}{*}{0,52} & \multirow{3}{*}{0,3323} \\
\hline & 48.30 & & & \\
\hline & 47.68 & & & \\
\hline
\end{tabular}

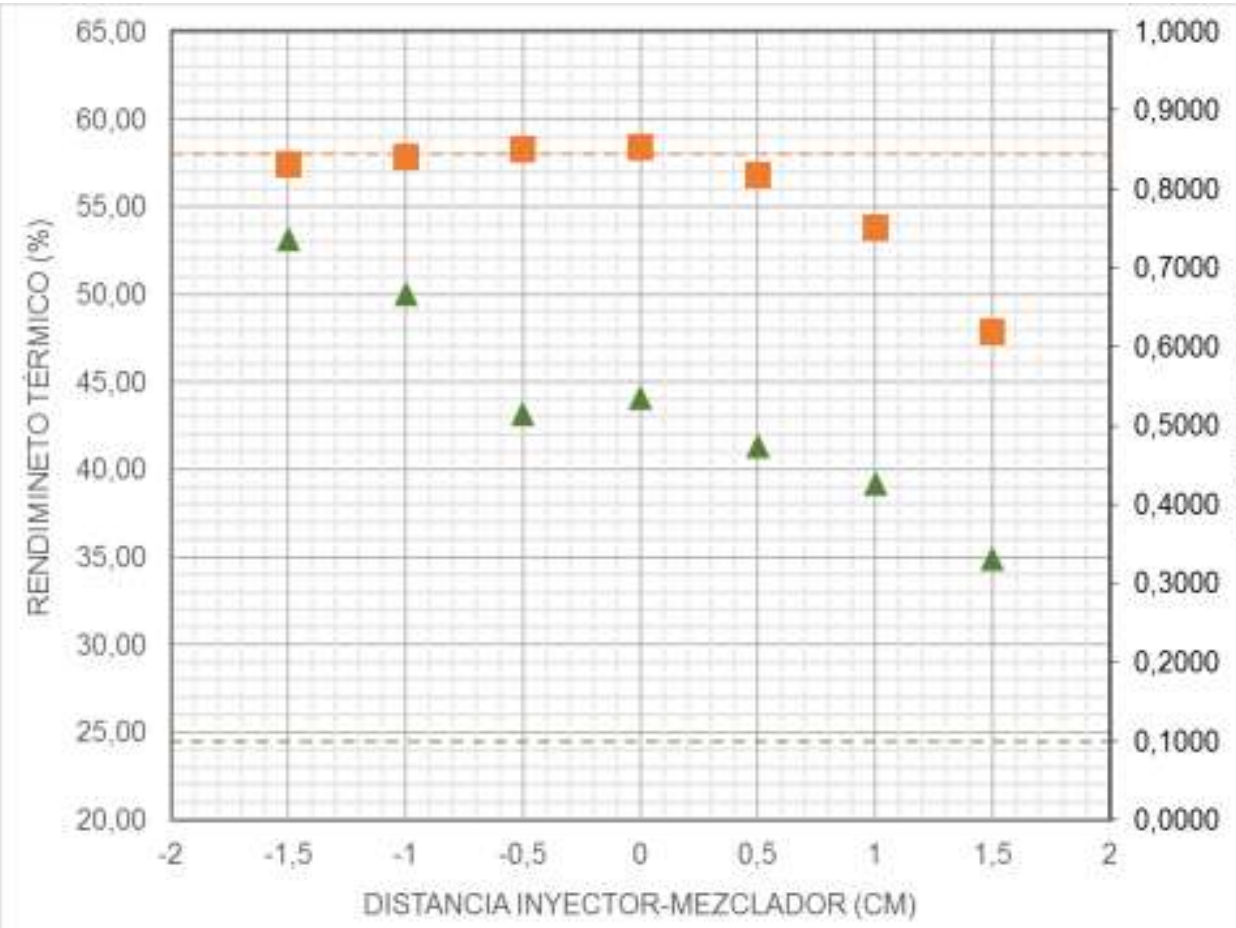

" Rendimiento Térmico Promedio con GLP-5 - - Límite Minimo de Rendimiento Térmico (58\%)

4 Emisiones COn generadas con GL.P-5 _ - - Límite Máximo de Emisiones COn (0.1\%)

Fig. 14: Rendimiento térmico y emisiones de $\mathrm{CO}_{\mathrm{n}}$ influenciados por la distancia "d" mezclador-inyector en el mezclador GLP-5 
En la Tabla 17 y en la Figura 15, se puede observar que al elevar la altura "h" entre la olla y la hornilla hasta un valor de $3,0 \mathrm{~cm}$, manteniendo una distancia $\mathrm{d}=-0,5 \mathrm{~cm}$ entre el inyector y el mezclador, el porcentaje de emisión se reduce a un nivel cercano a lo admitido, pero el rendimiento térmico se reduce hasta estar por debajo de límite admisible ( $55 \%$ aproximadamente). De manera que se podría resignar un cierto porcentaje de rendimiento para reducir el nivel de emisión de $\mathrm{CO}_{\mathrm{n}}$ a los al límite establecido por la norma NCh927/1.

Tabla 17: Rendimiento térmico y porcentaje de monóxido de carbono neutro usando el mezclador GLP-5, según la altura "h" entre la superficie inferior de la olla y la hornilla, manteniendo una distancia de 0,0 cm entre el inyector y el mezclador

\begin{tabular}{|c|c|c|c|c|}
\hline Altura "h" (cm) & $\begin{array}{c}\text { Mediciones de Rendimiento } \\
\text { Térmico (\%) }\end{array}$ & $\begin{array}{c}\text { Rendimiento Térmico } \\
\text { Promedio (\%) }\end{array}$ & $\begin{array}{c}\text { Incertidumbre Total } \\
(\%)\end{array}$ & $\begin{array}{c}\text { Monóxido de carbono } \\
\text { neutro (\%) }\end{array}$ \\
\hline \multirow{3}{*}{1} & 58,49 & \multirow{3}{*}{58,37} & \multirow{3}{*}{0,82} & \multirow{3}{*}{0,5364} \\
\hline & 59.31 & & & \\
\hline & 57,32 & & & \\
\hline \multirow{3}{*}{1,5} & 58,09 & \multirow{3}{*}{58,14} & \multirow{3}{*}{0,58} & \multirow{3}{*}{0,3765} \\
\hline & 57,99 & & & \\
\hline & 58,33 & & & \\
\hline \multirow{3}{*}{2} & 56,92 & \multirow{3}{*}{57,50} & \multirow{3}{*}{0,70} & \multirow{3}{*}{0,2868} \\
\hline & 58,25 & & & \\
\hline & 57,31 & & & \\
\hline \multirow{3}{*}{2,5} & 56,13 & \multirow{3}{*}{55,70} & \multirow{3}{*}{0,61} & \multirow{3}{*}{0,1558} \\
\hline & 55,71 & & & \\
\hline & 55,24 & & & \\
\hline \multirow{3}{*}{3} & 55,21 & \multirow{3}{*}{55,10} & \multirow{3}{*}{0,56} & \multirow{3}{*}{0,1310} \\
\hline & 55,23 & & & \\
\hline & 54,85 & & & \\
\hline
\end{tabular}

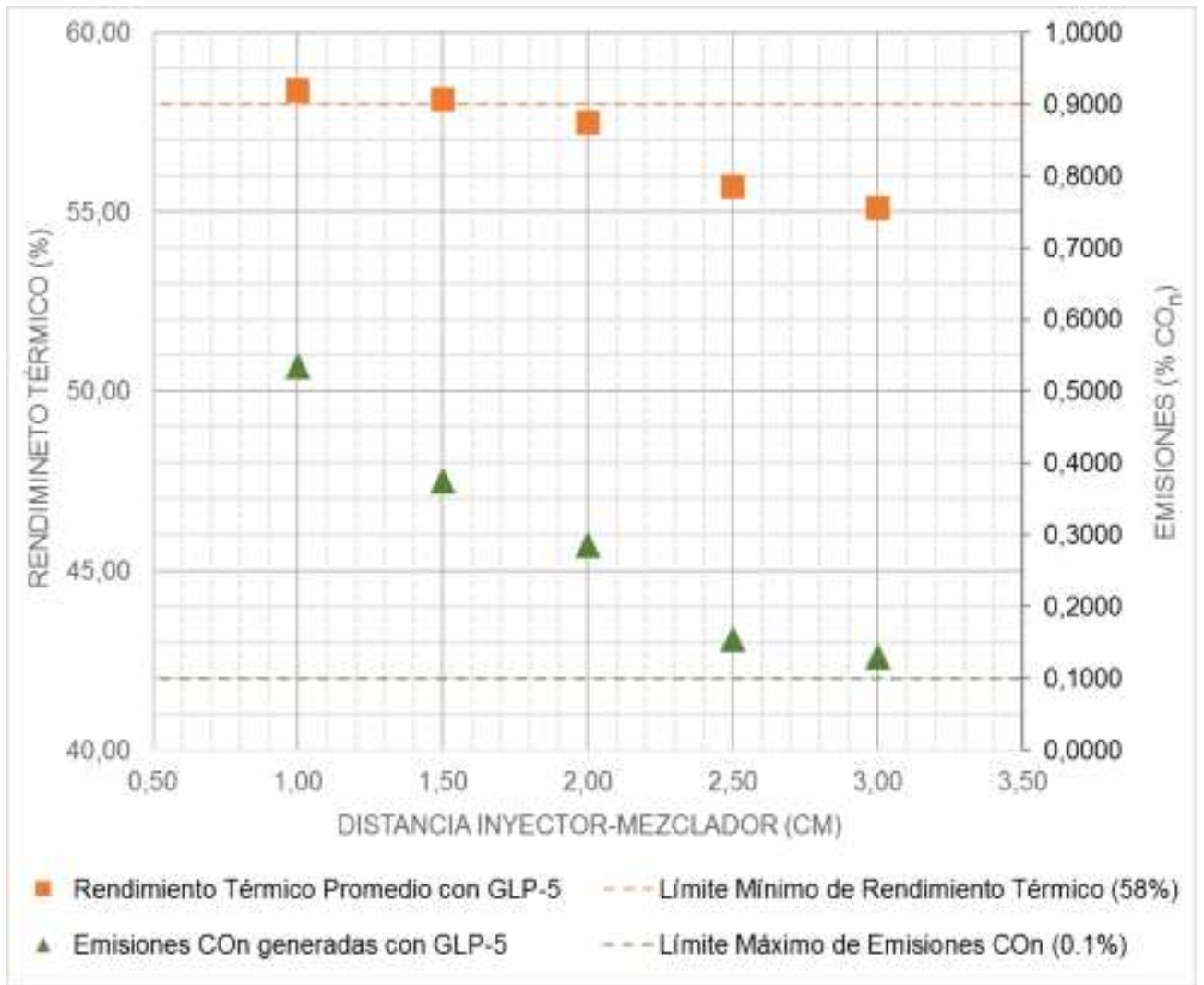

Fig. 15: Rendimiento térmico y emisiones de $\mathrm{CO}_{\mathrm{n}}$ influenciados por la altura "h" quemadorolla en mezclador GLP-5 


\section{Modelo GLP-6}

Como se puede observar en la Tabla 18 y en la Figura 16, en el mezclador GLP-6 se obtiene el mayor rendimiento térmico cuando el inyector está a una distancia de $0,0 \mathrm{~cm}$ con respecto al mezclador $(\mathrm{d}=0,0 \mathrm{~cm})$ y una altura entre el quemador y la superficie inferior de la olla de $1,0 \mathrm{~cm}(\mathrm{~h}=1,0 \mathrm{~cm})$, superando el mínimo valor de rendimiento térmico recomendado (58 \%) y el máximo valor promedio obtenido con el mezclador GLP-3, con el cual difiere solo en el ángulo de entrada de la sección convergente del mezclador (ver Tabla 6).

Tabla 18: Rendimiento térmico y porcentaje de monóxido de carbono neutro usando el mezclador GLP-6, según la distancia "d" del inyector con respecto al mezclador

\begin{tabular}{|c|c|c|c|c|}
\hline $\begin{array}{l}\text { Distancia "d" } \\
(\mathrm{cm})\end{array}$ & $\begin{array}{c}\text { Mediciones de Rendimiento } \\
\text { Térmico (\%) }\end{array}$ & $\begin{array}{c}\text { Rendimiento Térmico } \\
\text { Promedio (\%) }\end{array}$ & $\begin{array}{c}\text { Incertidumbre Total } \\
(\%)\end{array}$ & $\begin{array}{c}\text { Monóxido de carbono } \\
\text { neutro (\%) }\end{array}$ \\
\hline \multirow{3}{*}{0,0} & 56,85 & \multirow{3}{*}{56,82} & \multirow{3}{*}{0,56} & \multirow{3}{*}{0,5406} \\
\hline & 56,78 & & & \\
\hline & 56,84 & & & \\
\hline \multirow{3}{*}{0,5} & 56,27 & \multirow{3}{*}{56,22} & \multirow{3}{*}{0,56} & \multirow{3}{*}{0,4992} \\
\hline & 56,12 & & & \\
\hline & 56,27 & & & \\
\hline \multirow{3}{*}{1,0} & 55,05 & \multirow{3}{*}{55,57} & \multirow{3}{*}{0,66} & \multirow{3}{*}{0,4551} \\
\hline & 56,26 & & & \\
\hline & 55,39 & & & \\
\hline \multirow{3}{*}{1,5} & 54,55 & \multirow{3}{*}{54,74} & \multirow{3}{*}{0,55} & \multirow{3}{*}{0,4289} \\
\hline & 54,88 & & & \\
\hline & 54,78 & & & \\
\hline \multirow{3}{*}{2,0} & 52.13 & \multirow{3}{*}{52.46} & \multirow{3}{*}{0,69} & \multirow{3}{*}{0,3834} \\
\hline & 53.36 & & & \\
\hline & 51.90 & & & \\
\hline
\end{tabular}

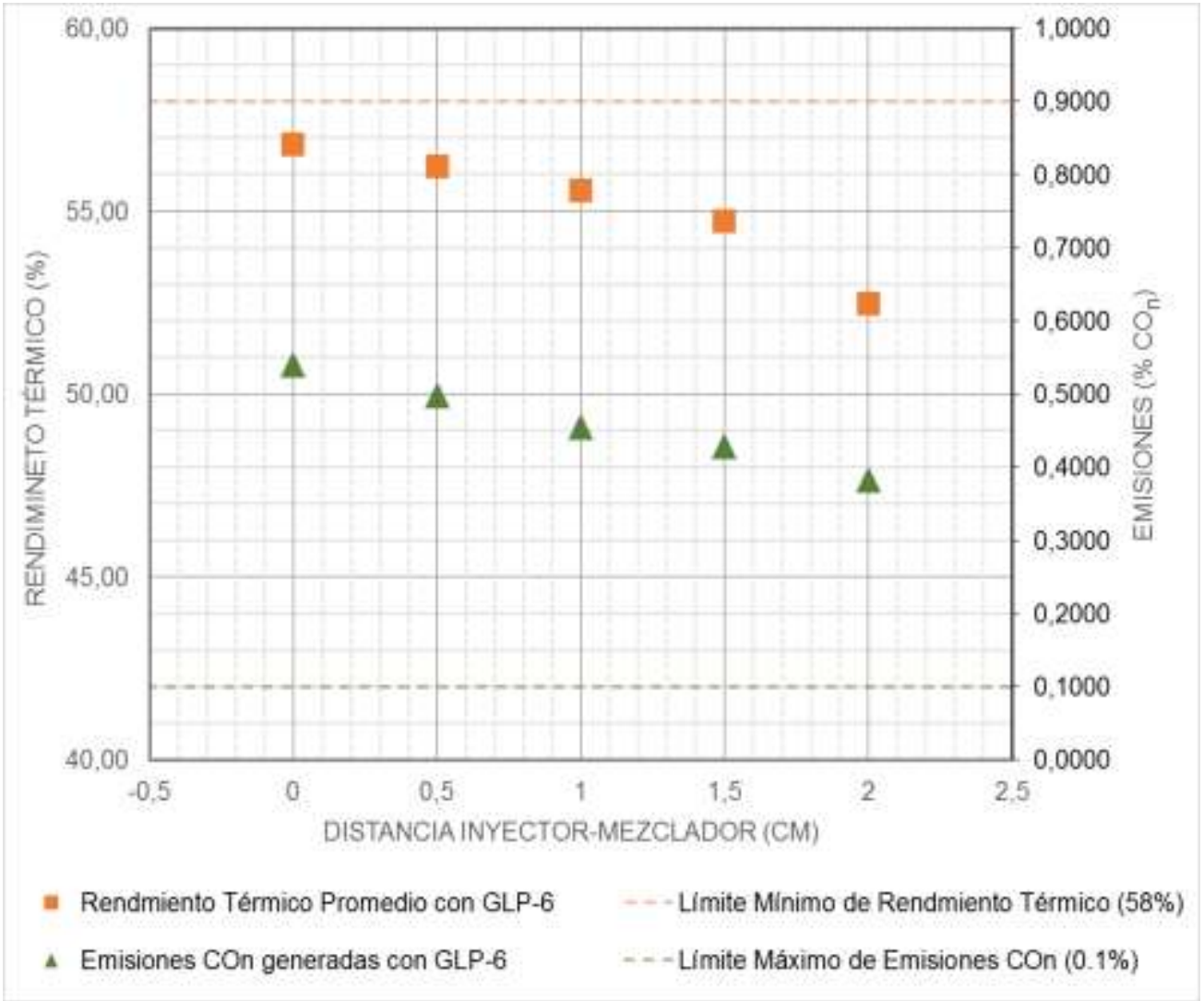

Fig. 16: Rendimiento térmico y emisiones de $\mathrm{CO}_{\mathrm{n}}$ influenciados por la distancia "d" mezclador-inyector en el mezclador GLP-6 
En la Tabla 19 y en la Figura 17, se observa que solo al elevar la altura entre el quemador y la superficie inferior de la olla a $2,5 \mathrm{~cm}(\mathrm{~h}=2,5 \mathrm{~cm})$, se obtiene una reducción significativa en el rendimiento térmico, tal como sucede con el modelo GLP-3. Mientras que en lo que respecta a la emisión de $\mathrm{CO}_{n}$, los valores conseguidos son mayores al límite establecido.

Tabla 19: Rendimiento térmico y porcentaje de monóxido de carbono neutro usando el mezclador GLP-6, según la altura "h" entre la superficie inferior de la olla y la hornilla, manteniendo una distancia de $0,0 \mathrm{~cm}$ entre el inyector y el mezclador

\begin{tabular}{|c|c|c|c|c|}
\hline Altura "h" (cm) & $\begin{array}{c}\text { Mediciones de } \\
\text { Rendimiento Térmico (\%) }\end{array}$ & $\begin{array}{c}\text { Rendimiento Térmico } \\
\text { Promedio (\%) }\end{array}$ & $\begin{array}{c}\text { Incertidumbre Total } \\
(\%)\end{array}$ & $\begin{array}{c}\text { Monóxido de carbono } \\
\text { neutro (\%) }\end{array}$ \\
\hline \multirow{3}{*}{1,0} & 56,85 & \multirow{3}{*}{56,82} & \multirow{3}{*}{0,56} & \multirow{3}{*}{0,5406} \\
\hline & 56,78 & & & \\
\hline & 56,84 & & & \\
\hline \multirow{3}{*}{1,5} & 56,76 & \multirow{3}{*}{56,47} & \multirow{3}{*}{0,58} & \multirow{3}{*}{0,4454} \\
\hline & 56,27 & & & \\
\hline & 56,39 & & & \\
\hline \multirow{3}{*}{2,0} & 56,32 & \multirow{3}{*}{56,14} & \multirow{3}{*}{0,56} & \multirow{3}{*}{0,4054} \\
\hline & 56,10 & & & \\
\hline & 56,01 & & & \\
\hline \multirow{3}{*}{2,5} & 54,60 & \multirow{3}{*}{54,21} & \multirow{3}{*}{0,64} & \multirow{3}{*}{0,2799} \\
\hline & 54,49 & & & \\
\hline & 53,54 & & & \\
\hline \multirow{3}{*}{3,0} & 53,03 & \multirow{3}{*}{53,37} & \multirow{3}{*}{0,56} & \multirow{3}{*}{0,1351} \\
\hline & 53,64 & & & \\
\hline & 53,44 & & & \\
\hline
\end{tabular}

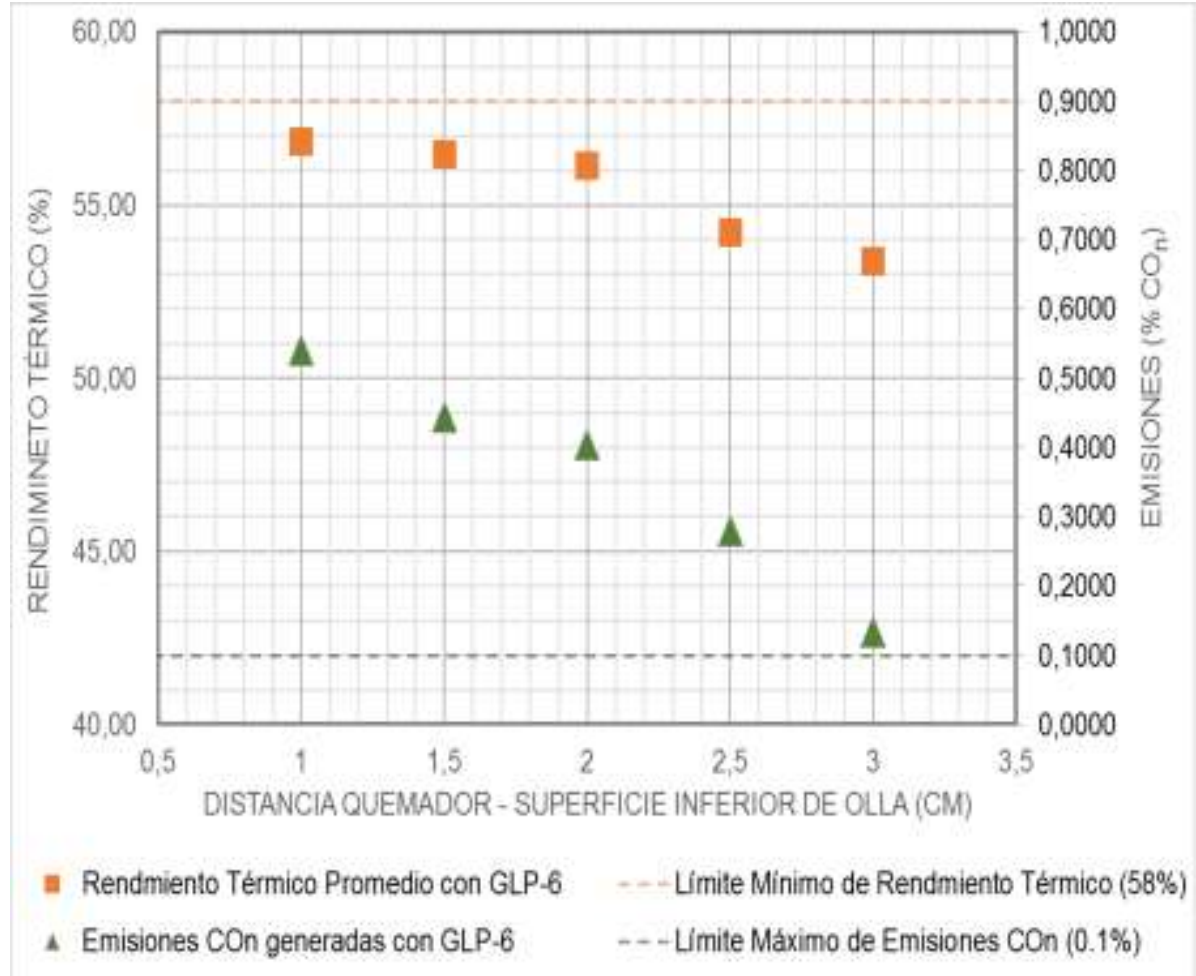

Fig. 17: Rendimiento térmico y emisiones de $\mathrm{CO}_{\mathrm{n}}$ influenciados por la altura " $\mathrm{n}$ " quemadorolla en mezclador GLP-6 


\section{CONCLUSIONES}

Nuestro trabajo de investigación ha contribuido a la literatura actual al ofrecer los resultados de un estudio experimental de seis mezcladores distintos para un quemador de premezcla de cocina doméstica a una potencia de 1,5 kW, mostrando la variación de rendimiento térmico y las emisiones de monóxido de carbono neutro a diferentes distancias entre el inyector y el mezclador, o el quemador y la superficie inferior de la olla con agua. Difiriendo de otras investigaciones en las que se centran en el diseño del quemador (Pantangi et al., 2011), $u$ otras en las que se analiza el rendimiento térmico de quemadores de cocinas domesticas convencionales (Gohil y Channiwala, 2011). Sin embargo, no se ha superado los rendimientos térmicos registrados en otras investigaciones (Olubiyi et al., 2014; Gohil y Channiwala, 2011). De manera que se ha obtenido información experimental de lo planteado por diversos autores, así como de los efectos que pueden generarse al modificar la forma geométrica del mezclador.

En lo que respecta a los diseños propuesto por los autores estudiados, al comparar los resultados de los ensayos de los mezcladores basados en los modelos matemático mostrados, específicamente del modelo GLP-1 (Jones, 1989) Y el modelo GLP-4 (Fulford, 1996), se puede comprobar que lo indicado por el autor Fulford (1996) permite el diseño de un mezclador con mejores resultados, obteniéndose un rendimiento térmico por encima del $58 \%$, pero un aumento en la cantidad emisiones de monóxido de carbono de 1.2 veces. Con las características del modelo GLP-5 (altura entre quemador y olla de 1,5 cm; distancia entre inyector y mezclador de $0 \mathrm{~cm}$ ) se encuentra el rendimiento más alto de 58,14\% mayor a lo establecido por norma técnica internacional.

En lo que respecta a los efectos en la geometría del diseño en el mezclador, según los ensayos experimentales, se llega a la conclusión de que no necesariamente la sección convergente es el principal motivo por el que el rendimiento térmico sea más alto, de manera que la longitud más amplia en la sección convergente del modelo GLP-5 no necesariamente es la principal causa de la obtención de un punto más alto de rendimiento térmico. Esto puede verse tras comparar los resultados del modelo GLP-1 y GLP-3, según los cuales se puede determinar que el aumento de la sección convergente no conlleva un crecimiento de los valores de rendimiento térmico, sino una leve reducción en los resultados.

Asimismo, la variación del ángulo de entrada, pasando de los $40^{\circ}$ usados en el modelo GLP-3, a los 13,9ำ del modelo GLP-6, permite mejorar los valores de rendimiento térmico; sin embargo, debido a la reducción de la abertura de entrada de aire primario, en la mezcla aire - gas combustible existe un mayor porcentaje de GLP, produciendo que las emisiones de monóxido de carbono se incrementen. Además, se comprueba que una reducción en el largo de la sección divergente puede elevar los niveles de rendimiento térmico, tal como se puede verificar al contrastar los resultados de los ensayos de los mezcladores GLP-1 y GLP-2.

En las emisiones de $\mathrm{CO}_{n}$, bajo ningún caso se pudo registrar niveles por debajo de lo requerido $(0,1 \%)$, encontrando el más cercano al utilizar el mezclador GLP-5 (0,1310\%); manteniendo una distancia entre el mezclador y el inyector de $0,0 \mathrm{~cm}(\mathrm{~d}=0,0 \mathrm{~cm})$, y una altura entre el quemador y la superficie inferior de la olla de $3,0 \mathrm{~cm}(h=3,0)$. Sin embargo, en todos los mezcladores se pudo percatar que al alejar la olla del quemador se podía reducir las emisiones, esto debido a que, a mayor distancia, la llama generada podía tener un mejor desarrollo, evitando así la formación de las llamadas puntas amarillas, aunque ello generaba el decrecimiento del rendimiento térmico.

Cabe destacar que las incertidumbres obtenidas de los rendimientos térmicos alcanzados son, en su mayoría, valores por debajo del 1,00\%, siendo la única excepción un caso del mezclador GLP-3 (ver Tabla 12). En general, los números calculados poseen un valor aproximado de $0,60 \%$, de modo que la dispersión de datos se mantiene similar en todos los mezcladores. Además, se puede reconocer en las Tablas 8-19 que la incertidumbre total se incrementa en los ensayos donde se varía la distancia inyector-mezclador (distancia "d"), de manera que se concluye que la principal fuente de incertidumbre en la estimación del rendimiento térmico es esta distancia.

Por último, cabe destacar que en la actualidad el Perú no cuenta con una norma técnica referente al rendimiento térmico en cocinas domiciliarias. Por lo tanto, este estudio permitió realizar un proyecto de norma técnica que fue anexado en los documentos solicitados al proyecto concursable de investigación básica y aplicada 157-FINCYT-2013.

\section{AGRADECIMIENTOS}

Se agradece a INNOVATE PERÚ que a través de los Proyectos Concursables de Investigación Básica y Aplicada 157-FINCYT-IA-2013 en cooperación con la Pontificia Universidad Católica del Perú financiaron la investigación. 


\section{REFERENCIAS}

Baukal, Jr., C. E., Industrial burners Handbook. CrC press, Nueva York, Estados Unidos (2003)

Bisso, J., Determinación de la Velocidad de Llama Laminar en un quemador de premezcla. Pontificia Universidad Católica del Perú (2015)

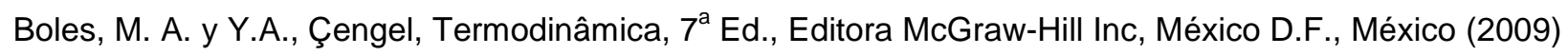

Cortés, C. L., Análisis numérico del flujo en un mezclador de aire combustible para un quemador atmosférico. Universidad Nacional Autónoma de México (2009)

European Commission, World energy, technology and climate policy outlook (en línea) (2003)

Fulford, D. D., Biogas Stove Design. Kingdom Bioenergy Ltd (1996)

Gohil, P. y S. Channiwala, Experimental Investigation Of Performance Of Conventional LPG Cooking stove, Fundamental, J. Thermal Science and Engg., I (1) (2011)

INN-CHILE, Artefactos de uso domésticos para cocinar, que utilizan combustibles gaseosos, Requisitos e inspección (2001)

Jones, H.R.N., The application of combustion principles to domestic gas burner design, Taylor \& Francis (1989)

Martinez, I., Procesos de combustión, Termodinámica Básica y Aplicada. Retrieved April 14, 2016, from https://goo.gl/RMcySh (1992)

Ministerio de Energía y Minas, Guía № 1. Elaboración de Proyectos de Guías de Orientación del Uso Eficiente de la Energía y de Diagnóstico Energético - Sector Residencial, 1-39. Disponible en: https://goo.gl/xvEKnR (2008a)

Ministerio de Energía y Minas, Usos y ventajas del gas natural en el sector residencial comercial, Ministerio de Energía y Minas, Dirección General de Hidrocarburos, Acceso: 14 de abril de 2016, Disponible en: https://goo.gl/p2g4HD (2008b)

Namkhat, A. y S. Jugjai, The Effect of Primary Air Preheat on the Primary Aeration of a Self- aspirating Burner, The First TSME International Conference on Mechanical Engineering (2010)

Olubiyi, O., A. Iheanyichukwu, M. Dauda y F. Olukayode, Design and Construction Of a Biogas Burner, Departamento de Ingeniería Mecánica, Ahmadu Bello University (2014)

OSINERGMING, Masificación del gas natural en el Perú: experiencia y perspectiva Perú (2014)

Pantangi, V.K., S.C. Mishra, P. Muthukumar y R. Reddy, Studies on porous radiant burners for LPG (liquefied petroleum gas) cooking applications (2011)

Rojas, F., Material del curso "Termodinámica 1" para Ciencias e Ingeniería, (P.U.C. del Perú, Ed.) (Pontificia) (2016)

Surange, J., N. Patil y A. Rajput, Performance Analysis of Burners used in LPG Cooking Stove-A Review, International Journal of Innovative Research in Science, Engineering and Technology (2014)

Thompson, D., J. Rivera y P. Pereyra, Manual de Laboratorio Computarizado de Física 1, Publicaciones P.U.C.P., Lima, Perú (2003) 
